\title{
中空纳米结构在表界面化学能源存储中的应用
}

\author{
毕如一卉, $a, b$ 毛丹 ${ }^{\dagger, a, c}$ 王江艳*,a,c 于然波*,b 王丹*,, , \\ ( ${ }^{a}$ 中国科学院过程工程研究所 生化工程国家重点实验室 北京 100190) \\ ${ }^{b}$ 北京科技大学 治金与生态工程学院 北京 100083) \\ ( ${ }^{c}$ 中国科学院大学 北京 100049)
}

\begin{abstract}
摘要 中空纳米结构因具有有效比表面积大、传输路径短、缓冲性能好等优势, 在能源转换和存储领域受到人们的广 泛关注, 本综述详细总结了中空纳米结构材料在以超级电容器为代表的表界面化学能源存储领域的研究进展. 首先介 绍了表界面化学能源存储的机理和挑战; 其次详细讨论了中空材料的微观结构参数对表界面化学能源存储装置性能 的影响; 然后系统概述了近年来研究者如何利用中空纳米结构解决表界面化学能源存储中的问题并优化电容器性能; 最后, 展望了中空纳米结构在表界面化学能源存储中面临的挑战和未来的发展方向.
\end{abstract}

关键词 中空纳米结构; 表界面; 超级电容器; 比电容

\section{Hollow Nanostructures for Surface/Interface Chemical Energy Storage Application}

\author{
$\mathrm{Bi}_{\text {Ruyi }}^{\dagger}{ }^{\dagger, a} \quad$ Mao, Dan ${ }^{\dagger, a, c} \quad$ Wang, Jiangyan ${ }^{*, a, c} \quad$ Yu, Ranbo*,b Wang, Dan ${ }^{*, a, c}$ \\ ( ${ }^{a}$ State Key Laboratory of Biochemical Engineering, Institute of Process Engineering, Chinese Academy of Sciences, \\ Beijing 100190, China) \\ $\left({ }^{b}\right.$ School of Metallurgical and Ecological Engineering, University of Science and Technology Beijing, Beijing 100083, China) \\ $\left({ }^{c}\right.$ University of Chinese Academy of Sciences, Beijing 100049, China)
}

\begin{abstract}
Hollow nanostructures garner tremendous interest in the area of energy conversion and storage, owning to its large surface area, facilitated transport path and good buffering capability. In this paper, we summarize the recent research on hollow nanostructures with controllable structure and morphology for surface/interface chemical energy storage. First, we introduce the charge storage mechanism and challenges of surface/interface chemical energy storage, mainly including supercapacitor. Subsequently, we discuss the influence of structure parameters of hollow nanostructures on the performance of surface/interface chemical energy storage device in detail. Afterwards, we systematically outline the recent applications of hollow nanostructures as electrode materials for supercapacitors. By adopting hollow nanostructures, the specific capacitance, cycle stability and rate capability of supercapacitors can be greatly improved. Finally, the emergent challenges and future development directions in hollow nanostructures for surface/interface chemical energy storage are provided.

Keywords hollow nanostructure; surface/interface; supercapacitor; specific capacitance
\end{abstract}

\section{1 引言}

全球经济的快速发展, 导致化石燃料短缺和环境污 染等问题日益严峻, 引起了人们对新型可再生能源的重 视 ${ }^{[1-4]}$. 尽管太阳能、风能、地热能等可再生清洁能源已 被广泛报道, 但是其不稳定性和间歇性限制了它们的商 业应用范围 ${ }^{[5,6]}$. 因而, 迫切需求一种先进且高效的能源 转换和存储装置来保证能源的平稳输出. 超级电容器和 二次电池作为两种主要的电化学储能器件能够缓解甚 至解决上述问题, 在许多领域得到了广泛的应用 ${ }^{[7,8]}$. 虽 然二次电池能量密度高 $\left(150 \sim 200 \mathrm{Wh} \cdot \mathrm{kg}^{-1}\right)$, 但其功率
密度较低 $\left(<1 \mathrm{~kW} \cdot \mathrm{kg}^{-1}\right)$ 、循环寿命较短 $(<1000$ 次 $)$ 、充 电时间长, 不能满足工业应用的需求 ${ }^{[9,10]}$. 相比之下, 超级电容器由于具有长的循环寿命 $\left(10^{4} \sim 10^{5}\right.$ 次 $)$ 和高的 功率密度 $\left(>10 \mathrm{~kW} \cdot \mathrm{kg}^{-1}\right)$ 等优点而备受关注 ${ }^{[11,12]}$. 此外, 其能量密度比传统电容器高 $1 \sim 2$ 个数量级, 兼具电容 器和电池的特性, 可以很好地填补传统电容器和二次电 池之间的空白. 超级电容器是常见的表面/界面电化学 储能器件, 其能量存储主要来自电极表面与近表面和电 极/电解液界面, 可以实现快速充放电. 高性能的超级电 容器, 不但需要足够大的比表面积以提供更多的电化学 活性位点, 还应当具有合适的孔结构以改善电极/电解

\footnotetext{
*E-mail: jywang@ipe.ac.cn; ranboyu@ustb.edu.cn; danwang@ipe.ac.cn

$\uparrow$ These authors contributed equally to this work.

Received June 8, 2020; published August 14, 2020.

Project supported by the National Natural Science Foundation of China (Nos. 51872024, 21820102002, 21590795, 51661165013) and the Scientific Instrument Developing Project of the Chinese Academy of Sciences (No. YZ201623).

项目受国家自然科学基金(Nos. 51872024, 21820102002, 21590795, 51661165013)和中国科学院科研装备研制项目(No. YZ201623)资助.
} 
液界面的离子传输. 因此, 具有多孔特征和高比表面积 的中空纳米材料被普遍认为是表界面化学能源存储的 理想材料 ${ }^{[13,14]}$.

中空纳米结构具有密度低、比表面积大、物质/电荷 传输路径短、缓冲性能好等特点 ${ }^{[15-20]}$. 由于其独特的结 构优势和高度可控的物理化学性质, 中空纳米结构在能 源存储和转换领域(二次电池、超级电容器、锂硫电池、 燃料电池、染料敏化太阳能电池) 已被广泛报道 ${ }^{[21-34]}$. 中 空纳米结构作为超级电容器电极材料具有以下优点: (1)中空纳米结构具有高的有效比表面积, 可以提供丰 富的电化学活性位点, 从而获得更高的比电容; (2)高的 孔隙率使得电解液进入中空结构材料的内部, 从而提高 电解液润湿性, 增加电极/电解液界面, 并且较薄壳壁可 以缩短离子和电子的传输路径, 从而提高反应速率; (3) 内部空腔结构可以缓冲充放电过程中体积的膨胀, 提高 材料循环稳定性, 且多壳层中空结构由于不同壳层互相 支撑，具有更好的机械性能.

近年来，尽管有许多优秀的综述报道了表界面化学 能源存储中电极材料的结构设计, 但是很少有综述文章 专门报道中空纳米结构材料在表界面化学能源存储中 的研究进展 ${ }^{[6-8,13]}$. 本综述首先介绍了表界面化学能源 存储的机理和存在的挑战; 其次重点讨论了采用中空纳 米结构用作表界面化学能源存储(主要指超级电容器)电 极材料, 以解决上述挑战并提高表界面化学能源存储性 能的最新进展; 最后, 展望了中空纳米结构材料应用于 表界面化学能源存储领域仍存在的技术挑战和未来的 研究方向. 这将有助于更好地理解表界面化学能源存储 的机理, 优化中空纳米结构, 以进一步提高表界面化学 能源存储装置的性能.

\section{2 表界面化学能源存储机理及挑战}

超级电容器是常见的表界面电化学储能装置, 根据 电荷存储机制的不同, 一般可分为双电层电容器和噟电 容电容器两种类型. 双电层电容器是最简单和商业化应

EDLC

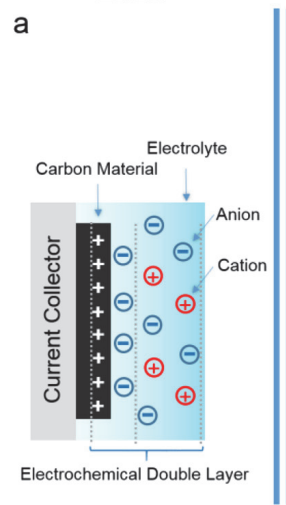

b

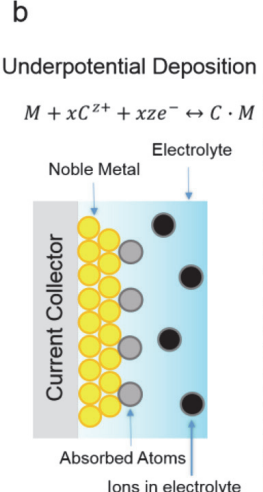

用最多的超级电容器, 主要通过电极表面的电荷形成双 电层来储存能源，其中电荷在电极材料表面聚积，电解 液中带相反电荷的离子在电解液侧富集(图 1a) ${ }^{[35,36]}$. 其 充放电过程是一个可逆的物理吸附/脱附过程，不发生 任何的法拉第氧化还原反应 ${ }^{[37,38]}$. 双电层电容材料通常 是具有多孔结构、比表面积较大、导电性和电化学稳定 性优异的碳基材料，如活性炭、碳纳米管和石墨烯 等[39-47].

赝电容电容器主要是通过在电极的表面/近表面区 域发生快速、可逆的电化学反应来实现能源存储. 噟电 容电容器在充放电过程由于发生法拉第反应，从而可以 达到比双电层电容器更高的比电容 ${ }^{[48]}$. 电极材料主要 包含过渡金属氧化物/硫化物/氢氧化物和导电聚合物 等 ${ }^{[49-52]}$. 根据噟电容电荷储能原理的不同, 可分为欠电 位沉积赝电容、氧化还原噟电容和插层赝电容三种类型

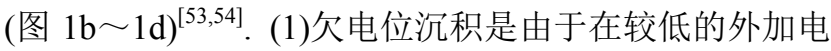
压下，离子 $\left(\mathrm{H}^{+}, \mathrm{Pb}^{2+}\right)$ 与贵金属表面之间存在很强的相 互作用力，被吸附在贵金属表面而形成单原子层 ${ }^{[55,56]}$. 由于贵金属成本较高以及工作电压窗口较窄等原因，欠 电位沉积很少能应用到能源存储中. (2)氧化还原噟电容 是一种典型的赝电容形式，基于电极材料的表面/近表 面发生电解液离子的吸附, 从而通过快速、可逆的氧化 还原反应来储存能量. 常见的氧化还原赝电容材料主要 包括 $\mathrm{RuO}_{2}, \mathrm{MnO}_{2}$, 导电聚合物等 ${ }^{[57-63]}$. (3)插层噟电容主 要是指离子插入到氧化还原活性材料的近表面而产生 的电容, 其电化学行为是介于超级电容器与二次电池之 间的过渡行为. 该过程基于电极材料晶格尺寸和纳米级 的颗粒尺寸，与电池中所涉及的发生晶体相变的插层反 应不同，该过程是不发生相变的法拉第过程. 插层赝电 容材料主要有 $\mathrm{Nb}_{2} \mathrm{O}_{5}, \mathrm{MoS}_{2}, \mathrm{MXene}$ 等 $^{[64-66]}$. 上述电容 器电极材料的赝电容特性是材料固有的, 与其晶粒的粒 径大小和形貌无关. 研究表明, 当一些电池型电极材料 具有某些特定的纳米结构或者尺寸减小到一定程度时, 其电化学行为发生显著的变化, 表现出噟电容特性,

\section{Pseudocapacitor}

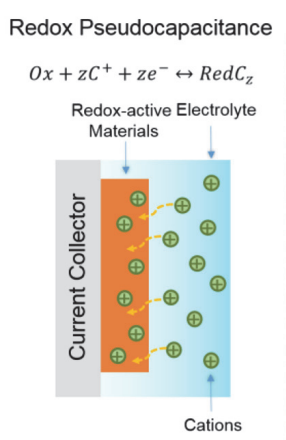

d

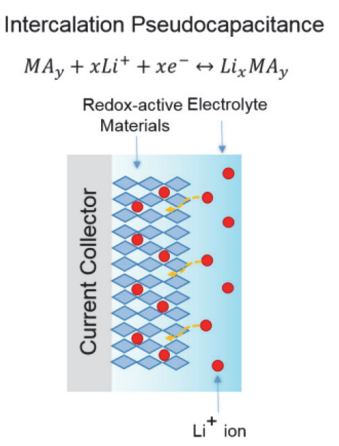

图 1 (a) 双电层电容器和 $(b \sim d)$ 不同类型赝电容电极的电荷存储机理图. 其中, (b) 欠电位沉积. (c) 氧化还原赝电容. (d) 离子插层赝电容 ${ }^{[36]}$. Figure 1 Schematics of charge-storage mechanisms for (a) an EDLC and (b d) different types of pseudocapacitive electrodes: (b) underpotential deposition, (c) redox pseudocapacitor, and (d) ion intercalation pseudocapacitor ${ }^{[36]}$. 
这种材料的赝电容特性是非固有的, 被认为非本征赝电 容材料 ${ }^{[11,36]}$. 非本征噟电容材料在尺寸较大时由于离子 存储过程中发生了相变, 因此并不会表现出赝电容. 非 本征噟电容的存在能够提高电池材料的功率密度, 这种 非本征赝电容是连接超级电容器和二次电池之间的桥 梁. 当材料尺寸纳米化, 降低到一定值后, 会显现出明 显的电容性特征 ${ }^{[67-69]}$. 例如, 块状 $\mathrm{LiCoO}_{2}$ 随着晶粒尺寸 的减小其放电平台逐渐变倾斜, 最终消失, 这时可以观 察到近似线性的充放电曲线(图 2) ${ }^{[70]}$. 这主要归因于表 面快速的氧化还原或非本征赝电容引起的低势垒电离 导致材料从电池型向噟电容型转变. 当材料尺寸达到纳 米尺度后, 会暴露更多的表面活性位点, 缩短了离子/电 子的传输路径, 从而提高了电容器的功率密度.

此外, 由两极分别为电容型材料与电池型材料组成 的混合电容器, 可以兼备能量密度高、功率密度大和循 环稳定性好的优势, 具有广阔的应用前景 ${ }^{[71,72]}$.

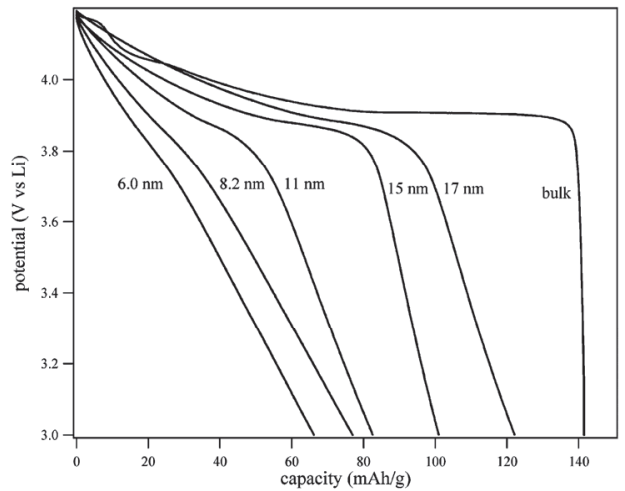

图 2 晶粒尺寸对 $\mathrm{LiCoO}_{2}$ 的锂化(放电)曲线的影响 ${ }^{[70]}$.

Figure 2 The effect of grain size on the lithiation (discharge) curve of $\mathrm{LiCoO}_{2}{ }^{[70]}$.

\section{3 中空材料的结构参数对表界面化学能源性能 的影响}

中空材料的微观结构参数(形貌、壳壁厚度、孔隙 率和壳层数, 图 3)会改变电极材料的电荷存储行为, 从 而对电容器的性能产生显著影响 ${ }^{[20,73-75]}$.

通过对合成过程进行精确的物理和化学调控可以 获得不同形貌的中空结构, 如球状、管状、立方体和多 面体等. 不同形貌的中空结构有着显著不同的物理化学 性能. 与其它非球形结构相比, 尽管球形结构具有相对 较低的比表面积, 但球形可以降低浆料中的表面张力, 保证电极活性材料在集流体上密集排布形成光滑且均 匀的电极片, 从而确保良好的导电性和循环稳定性 ${ }^{[76]}$.

中空材料的壳壁厚度和孔尺寸也对电容器性能有 重要影响. 通常情况下, 降低中空结构壳壁的厚度, 可 以提高比表面积和缩短离子/电子的传输路径, 从而提 高材料的能量密度和功率密度, 然而过薄的壳壁有可能 削弱材料的机械稳定性，通过优化中空结构壳壁的厚度， 可以同时保证良好的能源存储性能和机械稳定性. 中空
材料壳壁上的多孔结构既有利于增大材料的比表面积， 还可以促进电解液进入材料内部, 增大电解液与活性材 料的接触面积, 提高电容器的比电容和倍率性能. 此外, 多孔结构还能缩短离子扩散和电子迁移的路径，从而提 高电容器的快速充放电能力.

壳层数也能显著影响电容器性能. 在其他结构参数 一致的情况下, 中空结构的壳层数越多, 能提供的单位 体积比电容越高 ${ }^{[77-80]}$. 并且, 相对于中空单壳层结构, 中空多壳层结构(hollow multishelled structure, HoMS)因 外部壳层可以保护内部壳层，且多个壳层相互支撑，因 而具有更好的机械稳定性和电化学循环稳定性.

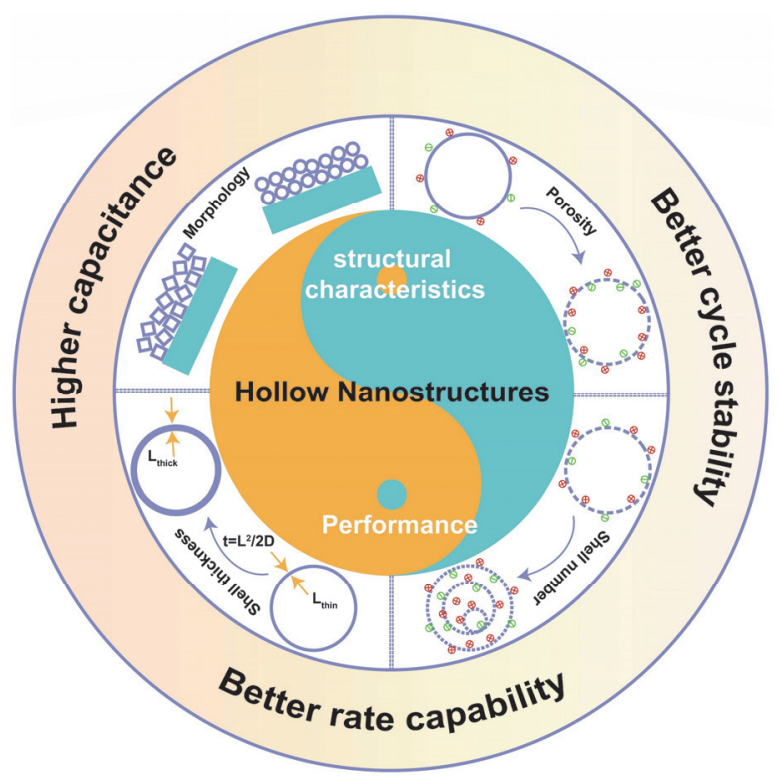

图 3 中空材料的结构参数对表界面化学能源性能的影响.

Figure 3 Scheme showing the influence of structural characteristics of hollow nanostructures on performance of surface/interface chemical energy.

\section{4 中空纳米结构在双电层电容材料中的应用}

碳材料用作双电层电容器的电极材料, 需要满足以 下要求: 足够大的比表面积、良好的导电性能以及合适 的孔尺寸以便于电解液的充分浸润 ${ }^{[81]}$. 目前使用最多 的碳材料主要有无定形活性炭(active carbon, AC)、石墨 烯和碳纳米管等 ${ }^{[82-86]}$. 目前，人们主要通过开发具有合 适孔径的中空碳材料来增大材料的比表面积, 以及对材 料表面进行修饰来改善性能.

Tan 等 ${ }^{[87]}$ 利用超交联微孔聚合物制备了中空微孔有 机胶囊，然后通过简单的碳化处理合成了无定形中空碳 球(图 4a). 通过优化超交联和碳化条件等合成参数可以 精确地控制壳层的厚度、空腔的大小和孔隙形貌. 用 15\%含量的二乙烯基苯前驱体在 $700{ }^{\circ} \mathrm{C}$ 下碳化后形成 的中空碳球组装成对称超级电容器, 在 $6 \mathrm{~mol} \cdot \mathrm{L}^{-1} \mathrm{KOH}$ 溶液中, 可提供 $5.4 \mathrm{Wh} \cdot \mathrm{kg}^{-1}$ 的高能量密度, 功率密度为 $1950 \mathrm{~W} \cdot \mathrm{kg}^{-1}$. 在 $50 \mathrm{mV} \cdot \mathrm{s}^{-1}$ 的扫速下, 循环 10000 圈后 
电容保持率在 $95.4 \%$ 以上(图 4b,c). 优异的电化学性能 主要得益于其独特的中空多孔结构增大了材料的比表 面积, 缩短了电子/离子的传输路径. 为了优化电极储能 性能，杂原子掺杂是一种常用的方法 ${ }^{[88-91]}$. Lu 等 ${ }^{[92]}$ 利用 可控的多界面转化方法(水热法、碳化处理和化学刻蚀)
成功地制备了形状尺寸均匀的氮掺杂中空多壳层碳微 球(图 4d). 通过调节反应时间能够控制壳层数量, 改变 碳源种类可以控制壳层的厚度. 氮掺杂的介孔碳壳为电 荷存储增加了反应活性位点，提高了导电能力. 合成的 氮掺杂的双层碳微球具有较高的比表面积 $2869.7 \mathrm{~cm}^{2}$ • a
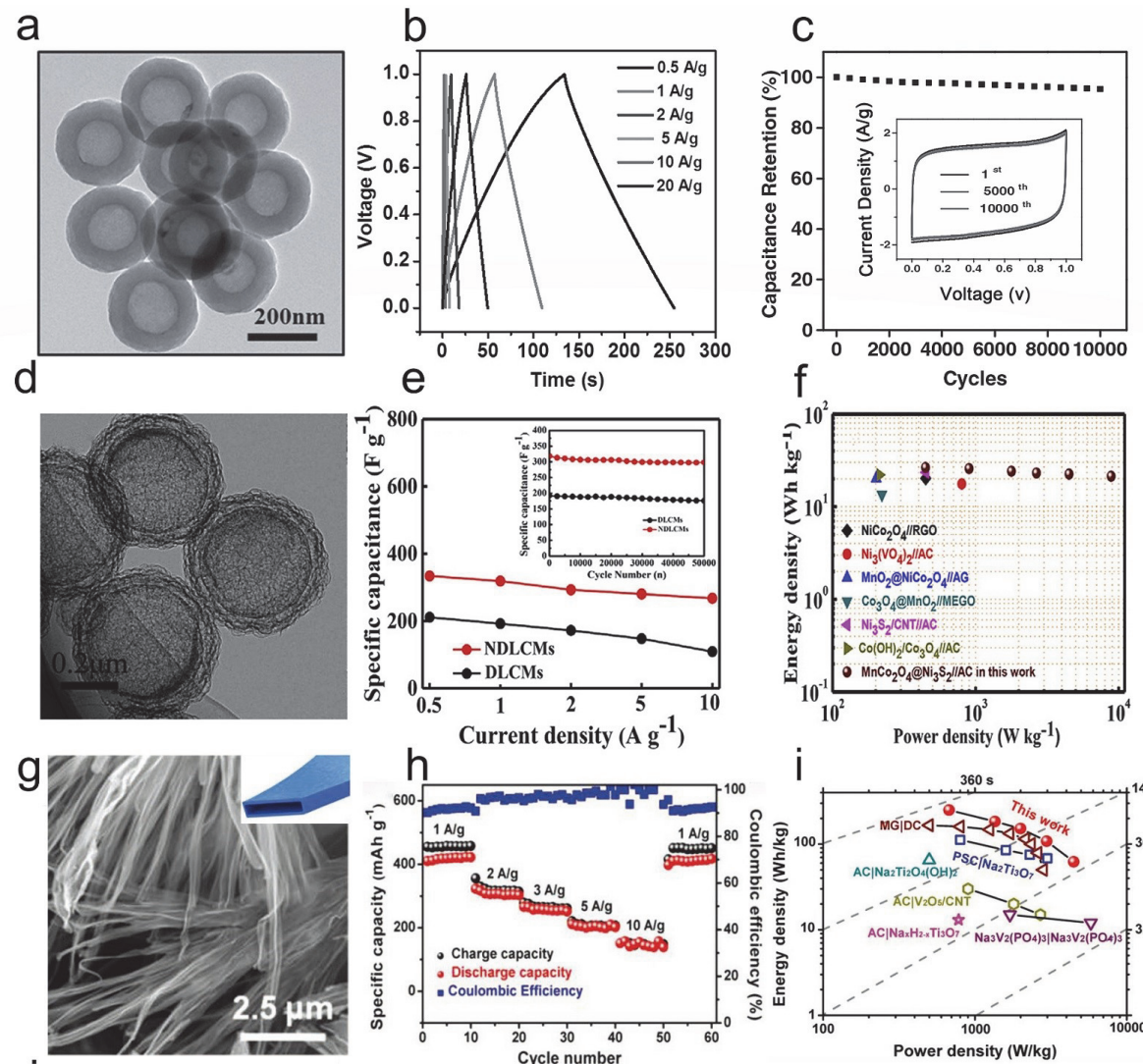

i
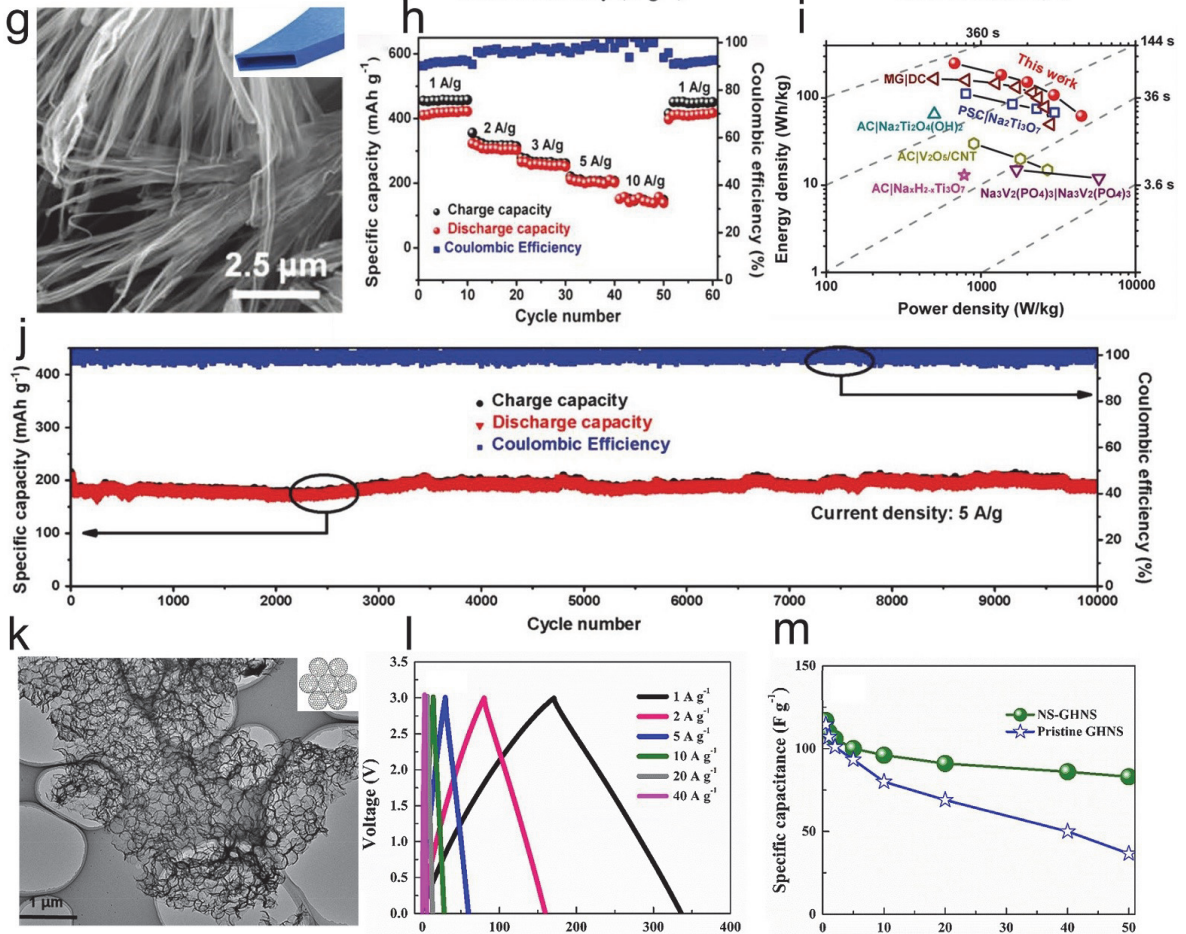

Power density (W/kg)

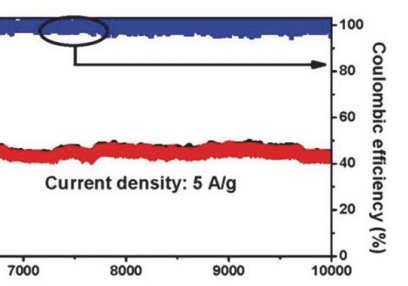

$\mathrm{m}$
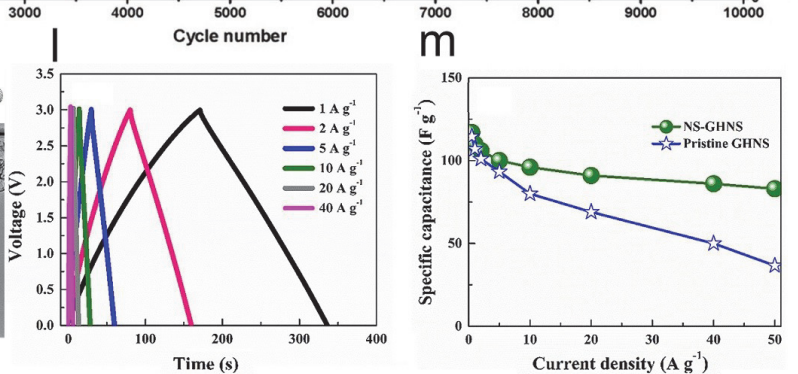

图 4 中空碳球的(a) TEM 图; (b) 充放电曲线; (c) 组装成对称超级电容器后在 $50 \mathrm{mV} \cdot \mathrm{s}^{-1}$ 扫速下的循环性能曲线, 插图为所选圈数的 CV 曲线 ${ }^{87]}$. 氮掺杂的双层碳微球 (d) TEM 图; (e) 在 $1 \mathrm{~A} \cdot \mathrm{g}^{-1}$ 电流密度下的循环曲线; (f) 组装成对称超级电容器的 Ragone 图 ${ }^{[92]}$. 氮硫共掺杂的中空碳纳米带 (g) SEM 图; N/S-HCNs | Sn 钠离子电容器的(h) 倍率性能; (i) Ragone 图; (j) 循环曲线 ${ }^{[3]}$. 氮和硫掺杂的石墨烯中空纳米球 (k) TEM 图; 组装成双 电层电容器的(1) 充放电曲线; (m) 倍率性能图 ${ }^{[94]}$.

Figure 4 (a) TEM images of hollow carbon spheres (HCS). (b) GCD curves of HCS. (c) Cycling stability of HCS based-symmetric SCs at a scan rate of $50 \mathrm{mV} \cdot \mathrm{s}^{-1}$ and (insets) CV curves of the selected cycles ${ }^{[87]}$. (d) TEM of the nitrogen doped double-layer carbon hollow microspheres (NDLCMs). (e) The cycling curves of NDLCMs at a current density of $1 \mathrm{~A} \cdot \mathrm{g}^{-1}$. (f) Ragone plot of the assembled symmetric supercapacitor ${ }^{[92]}$. (g) SEM of the N/S codoped hollow carbon nanobelts after the whole calcination approach. (h) The rate performance, (i) Ragone plots, (j) cycling stability of the N/S-HCNs $\mid$ Sn SIC ${ }^{[93]}$. (k) TEM images of graphene hollow nanospheres with dual heteroatoms (nitrogen and sulfur, NS-GHNS). (l) Charge/discharge profiles, (m) rate performances of NS-GHNS EDLC ${ }^{[94]}$. 
$\mathrm{g}^{-1}$ 和较大的孔体积 $8.08 \mathrm{~cm}^{3} \cdot \mathrm{g}^{-1}$. 氮掺杂的双层碳微球 能够提供 $318.5 \mathrm{~F} \cdot \mathrm{g}^{-1}$ 的比电容, 即使在 $1 \mathrm{~A} \cdot \mathrm{g}^{-1}$ 电流密 度下循环 50000 圈, 电容仍能保持 $93.7 \%$ (图 4e). 组装成 对称超级电容器, 在 $1 \mathrm{~mol} \cdot \mathrm{L}^{-1} \mathrm{Na}_{2} \mathrm{SO}_{4}$ 中性电解液中表 现出优异的电容器性能(图 4f). Tang 等 ${ }^{[93]}$ 通过自模板法 合成出氮硫共掺杂的中空碳纳米带材料(图 4g). 这种具 有高比表面积的中空纳米结构使得材料具有良好的吸 附/脱附能力, 从而加快了反应速率. 此外, 氮硫共掺杂 还贡献了额外的赝电容, 提高了材料的总电容. 用锡箔 作阳极，氮硫共掺杂的中空碳纳米带作阴极, $\mathrm{NaPF}_{6}$ 的 碳酸盐溶液为电解液, 组装成的钠离子电容器具有较高 的比电容和快速充放电能力(图 $4 \mathrm{~h}$ ). 在 $1 \mathrm{~A} \cdot \mathrm{g}^{-1}$ 下具有 $400 \mathrm{mAh} \cdot \mathrm{g}^{-1}$ 较高的比容量; 当电流密度升到 $10 \mathrm{~A} \cdot \mathrm{g}^{-1}$ 时, 放电比容量仍能达到 $155 \mathrm{mAh} \cdot \mathrm{g}^{-1}$. 此外, 该混合电 容器还表现出较高的能量密度、功率密度以及优异的循 环稳定性(图 4i, 4j).

近日, Lee 等 ${ }^{[94]}$ 利用牺牲模板法设计出氮和硫共掺 杂的石墨烯中空纳米球 (图 4k). 这种中空纳米结构提高 了石墨烯的表面利用率, 增加了活性位点的数量, 并缩 短了离子的扩散路径, 有助于实现优异的电容性行为. 氮和硫原子的掺杂有利于提高电化学活性, 增强电极表 面的电解液润湿性. 如图 41 所示, 氮硫掺杂的和未掺杂 的石墨烯中空球在 $0.5 \mathrm{~A} \cdot \mathrm{g}^{-1}$ 下的比电容分别 117 和 115 $\mathrm{F} \bullet \mathrm{g}^{-1}$, 相差不大. 但是, 当电流密度升至 $50 \mathrm{~A} \cdot \mathrm{g}^{-1}$ 时, 其电容分别保持初始值的 $71 \%$ 和 32\%(图 4m). 显然, 杂 原子掺杂的石墨烯中空球倍率性能优于未掺杂的, 这主 要归因于氮和硫的掺杂有利于离子的扩散以及改善电 子转移动力学.

\section{5 中空纳米结构在噟电容材料中的应用}

\section{1 氧化物}

与碳材料相比, 金属氧化物, 特别是具有多种氧化 还原态的金属氧化物表现出更高的比电容，显示出巨大 的潜力 ${ }^{[95-101]}$.

Wang 等 ${ }^{[102]}$ 采用次序模板法(sequential templating approach, STA), 以碳球为模板, 通过调节前驱体溶液的 $\mathrm{pH}$ 值合成了 $\mathrm{Mn}_{2} \mathrm{O}_{3}$ 多壳层中空球用作超级电容器电极 (图 5a, 5b). 三壳层 $\mathrm{Mn}_{2} \mathrm{O}_{3}$ 中空球在不同扫速下的循环 伏安(cyclic voltammogram, CV)曲线形状都保持得很好, 表明其具有理想的电容行为和优异的倍率性能 (图 $5 \mathrm{c}$ ). 在相同电流密度下, 单、双、三壳层 $\mathrm{Mn}_{2} \mathrm{O}_{3}$ 中空球的比 电容均比 $\mathrm{Mn}_{2} \mathrm{O}_{3}$ 纳米颗粒高, 体现出中空纳米结构的优 势. 此外, 三壳层 $\mathrm{Mn}_{2} \mathrm{O}_{3}$ 中空球在 $1 \mathrm{~A} \cdot \mathrm{g}^{-1}$ 下循环 2000

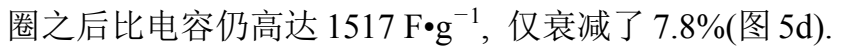

这主要归因于中空多壳层结构的外壳对内壳的保 护作用, 减缓了内壳的电化学溶解, 提高了结构稳定性. 之后, Chen 等 ${ }^{[103]}$ 通过水热强化阴离子吸附成功制备了 价态、壳层数、壳壁表面粗䊁度可调的 $\mathrm{MnO}_{2}$ 多壳层中
空球(图 $5 \mathrm{e} \sim 5 \mathrm{~h}$ ). 四壳层 $\mathrm{MnO}_{2}$ 中空球表现出高的比电 容和优异倍率性能, 当电流密度为 $0.5 \mathrm{~A} \cdot \mathrm{g}^{-1}$ 时, 比电容 为 $1457 \mathrm{~F} \cdot \mathrm{g}^{-1}$, 即使升高至 $10 \mathrm{~A} \cdot \mathrm{g}^{-1}$ 时, 仍高达 $1037 \mathrm{~F} \bullet$ $\mathrm{g}^{-1}$.

在各种电极材料中, $\mathrm{Co}_{3} \mathrm{O}_{4}$ 由于理论比电容和氧化 还原活性高, 被公认是最具有发展前景的噟电容电极材 料之一. 因此, Wang 等 ${ }^{[104]}$ 以碳球为模板, 通过控制吸 附条件成功获得了 $\mathrm{Co}_{3} \mathrm{O}_{4}$ 中空多壳层微球. 三壳层和 四壳层 $\mathrm{Co}_{3} \mathrm{O}_{4} \mathrm{HoMSs}$ 的壳层表面有许多褶皱，这有利 于比表面积的增大(图 5i,j). 三壳层 $\mathrm{Co}_{3} \mathrm{O}_{4} \mathrm{HoMSs}$ 由于 具有较大的比表面积和较薄的壳壁厚度, 表现出最优异 的电化学性能(图 $5 \mathrm{k}, 5 \mathrm{l}$ ), 在 $0.5 \mathrm{~A} \cdot \mathrm{g}^{-1}$ 下比电容最高达 $688.2 \mathrm{~F} \cdot \mathrm{g}^{-1}$, 并且在电流密度升至 $10 \mathrm{~A} \cdot \mathrm{g}^{-1}$ 时, 仍然具 有 $89.8 \%$ 电容保持率, 在 $2 \mathrm{~A} \cdot \mathrm{g}^{-1}$ 下循环 2000 圈后还能 保持初始电容的 $93.2 \%$. 这主要得益于 $\mathrm{Co}_{3} \mathrm{O}_{4} \mathrm{HoMSs}$ 多 个壳层之间可以相互支撑, 缓冲氧化还原反应带来的形 变应力, 因而增强了结构稳定性.

$\mathrm{Nb}_{2} \mathrm{O}_{5}$ 是典型的插层赝电容材料, 是混合离子电容 器的良好候选电极材料, 近年来得到了广泛关注 ${ }^{[105]}$. Wang 课题组 ${ }^{[106]}$ 采用 STA 法成功合成了壳层数可控的 $\mathrm{Nb}_{2} \mathrm{O}_{5}$ 中空多壳结构(图 $5 \mathrm{~m}$ ). 从图 $5 \mathrm{n}$ 可以得知, 其电荷 存储机制是电容行为占主导. 将三壳层 $\mathrm{Nb}_{2} \mathrm{O}_{5} \mathrm{HoMSs}$ 与活性炭组装成锂离子电容器, 其 $\mathrm{CV}$ 曲线表现出近矩 形的形状，表明了电荷存储机制为电解液中的 $\mathrm{PF}_{6}{ }^{-}$在 阴极侧发生的快速物理吸脱附和阳极侧发生的 $\mathrm{Li}^{+}$较慢 插层反应相结合. 该锂离子电容器兼具高的能量密度和 功率密度, 并且具有优异的循环稳定性(图 5o, 5p). 这主 要由于 HoMSs 的独特结构提供了丰富的反应位点, 降 低了电子输运阻力, 提高了离子输运的扩散速率.

二元过渡金属氧化物 $\mathrm{CoFe}_{2} \mathrm{O}_{4}$ 由于具有高比电容、 高效的电催化行为和优异的化学稳定性被认为是极具 潜力的超级电容器电极材料, 但是其电化学性能仍有待 提高 ${ }^{[107]}$. $\mathrm{Li}$ 等 ${ }^{[108]}$ 以环糊精为模板, 通过一步法制备了 $\mathrm{CoFe}_{2} \mathrm{O}_{4}$ 多壳层中空球. 合成过程中, 通过控制水和乙 醇的比例, 能够控制中空球的壳层数和内部结构(图 $5 \mathrm{q}$, 5r). 这种中空纳米结构不仅缩短了电解液离子的传输 路径, 还为氧化还原反应提供了更多的活性比表面积. 从图 $5 \mathrm{~s}, 5 \mathrm{t}$ 中可以看出, 三壳层 $\mathrm{CoFe}_{2} \mathrm{O}_{4} \mathrm{HoMSs}$ 具有优 异的倍率性能和循环稳定性.

\section{2 硫化物}

作为一类新型的储能材料, 和金属氧化物相比, 金 属硫化物具有电子电导率高、成本低、易于制造和低毒 性等优点而受到广泛的研究 ${ }^{[109-112]}$. 此外, 金属硫化物 的导电性可以通过调控硫的含量来调节，在金属种类和 价态一定的条件下, 硫化物中硫的含量越大, 理论电容 越高、氧化还原电位越低、导电性越好, 可预期的性能 越好. 
a

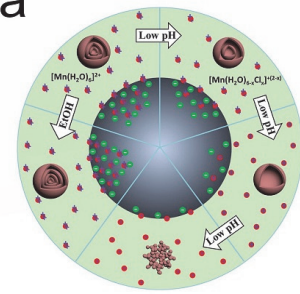

e

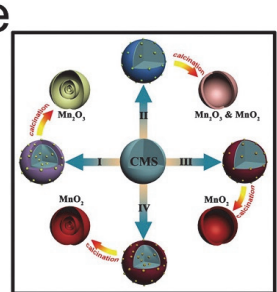

i
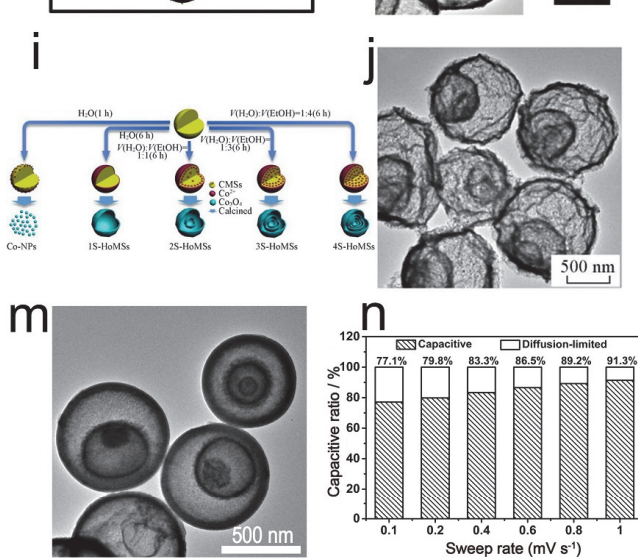

q

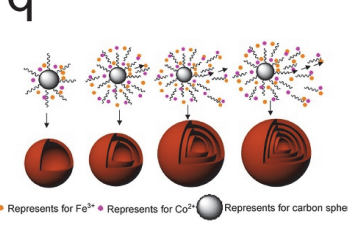

b

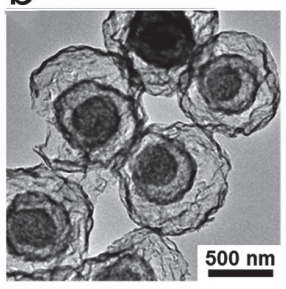

$\mathrm{f}$

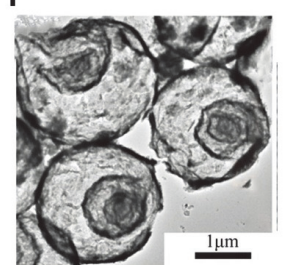

$\mathrm{n}$
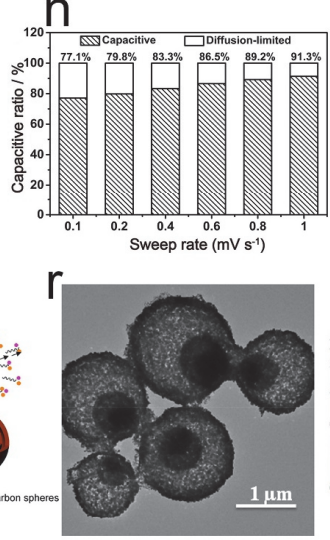

C

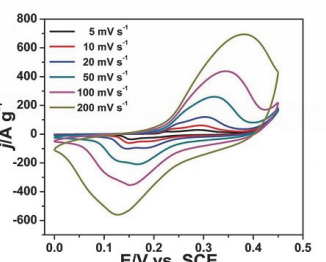

$\mathrm{g}$


$s$

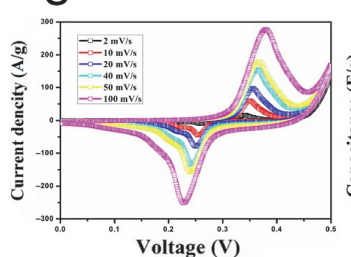

d

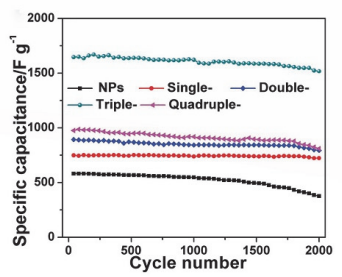

$\mathrm{h}$
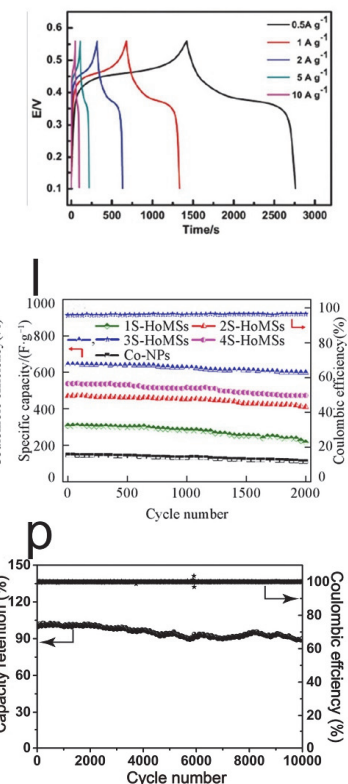

$\mathrm{t}$

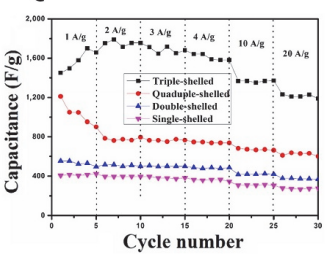

图 5 (a) $\mathrm{Mn}_{2} \mathrm{O}_{3}$ 纳米颗粒和多壳层中空球的合成机理图; (b) 三壳层 $\mathrm{Mn}_{2} \mathrm{O}_{3}$ 中空球的 $\mathrm{TEM}$ 图; (c) CV 图; (d) $\mathrm{Mn}_{2} \mathrm{O}_{3}$ 在 $1 \mathrm{~A} \cdot \mathrm{g}^{-1}$ 下循环性能曲线 [102]. (e) 采用不同锰源, 选择普通离子吸附法(线路 I, II 和 III)和水热强化吸附法(线路 IV)的机理对比; (f) 四壳层 $\mathrm{MnO}_{2}$ 中空球的 TEM 图; (g) CV 图; (h) 充放电曲线 ${ }^{[103]}$. (i) $\mathrm{Co}_{3} \mathrm{O}_{4}$ 多壳层中空球和纳米颗粒的合成过程; (j) 三壳层 $\mathrm{Co}_{3} \mathrm{O}_{4}$ 中空球的 TEM 图; (k) $\mathrm{Co}_{3} \mathrm{O}_{4}$ 多壳层中空球和纳米颗粒的比电容和

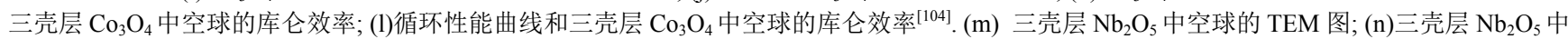

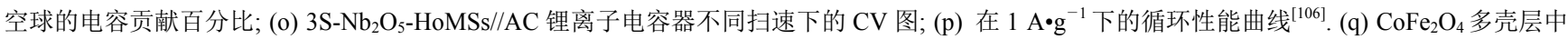
空球合成示意图; (r) 三壳层 $\mathrm{CoFe}_{2} \mathrm{O}_{4}$ 中空球的 TEM 图; (s) CV 图; (t) 倍率性能图 ${ }^{[108]}$.

Figure 5 (a) Scheme of synthesis mechanism for $\mathrm{Mn}_{2} \mathrm{O}_{3}$ nanoparticles and multishelled hollow microspheres. (b) TEM image of triple-shelled $\mathrm{Mn}_{2} \mathrm{O}_{3}$ hollow microspheres. (c) $\mathrm{CV}$ curves of triple-shelled $\mathrm{Mn}_{2} \mathrm{O}_{3}$ hollow microspheres. (d) Cyclic stability of $\mathrm{Mn}_{2} \mathrm{O}_{3}$ samples ${ }^{[102]}$. (e) Conventional solution adsorption method under ambient conditions (Routes I, II and III) and the hydrothermal intensification method (Route IV) with various Mn precursors. (f) TEM images of quadruple-shelled $\mathrm{MnO}_{2}$ hollow microspheres. (g) $\mathrm{CV}$ curves of quadruple-shelled $\mathrm{MnO}_{2}$ hollow microspheres. (h) Galvanostatic charge/discharge curves of quadruple-shelled $\mathrm{MnO}_{2}$ hollow microspheres at different current densities ${ }^{[103]}$. (i) Synthesis processes for 1S-, 2S-, 3S-, 4S-HoMSs and Co-NPs. (j) TEM images of 3S-HoMSs. (k) Specific capacitances of 1S-, 2S-, 3S- and 4S-HoMSs and Co-NPs and Coulombic efficiency of 3S-HoMSs at different current densities. (1) Cyclic stability of 1S-, 2S-, 3S-, 4S-HoMSs and Co-NPs and corresponding Coulombic efficiency of $3 \mathrm{~S}-\mathrm{HoMSs}$ at a current density of $2 \mathrm{~A} / \mathrm{g}^{[104]}$. (m) TEM micrographs of $3 \mathrm{~S}-\mathrm{Nb}_{2} \mathrm{O}_{5}$-HoMSs. (n) Capacitive contributions of $3 \mathrm{~S}-\mathrm{Nb}_{2} \mathrm{O}_{5}$-HoMSs. (o) CV curves at different scan rates, (p) Cycling performance at $1 \mathrm{~A} \cdot \mathrm{g}^{-1}$ of $3 \mathrm{~S}_{-}-\mathrm{Nb}_{2} \mathrm{O}_{5}-\mathrm{HoMSs} / / \mathrm{AC}$ LICs device ${ }^{[106]}$. (q) Illustration showing enhancement of the pore infiltration and complexing ability of $\mathrm{Fe}^{3+}, \mathrm{Co}^{2+}$ in infusion solutions of increasing ethanol content. ( $\mathrm{r}$ ) TEM images of $\mathrm{CoFe}_{2} \mathrm{O}_{4}$ triple-shelled hollow microspheres. (s) Cyclic voltammograms of $\mathrm{CoFe}_{2} \mathrm{O}_{4}$ triple-shelled hollow microspheres. (t) Rate-capability test for $\mathrm{CoFe}_{2} \mathrm{O}_{4}$ spheres at various current densities $(1 \sim 20 \mathrm{~A} / \mathrm{g})^{[108]}$.

$\mathrm{Li}$ 等 ${ }^{[113]}$ 以 $\mathrm{NiO}$ 中空球为前驱体, 通过控制硫化条 件制备了 $\mathrm{NiS}_{2}$ 多壳层中空球. 实验结果表明, 相对于纳 米颗粒和较少层数的中空球, 四壳层 $\mathrm{NiS}_{2}$ 中空球表现 出更好的电化学行为, 在 $5 \mathrm{~A} \cdot \mathrm{g}^{-1}$ 电流密度下, 其比容量
为 $216 \mathrm{mAh} \cdot \mathrm{g}^{-1}$, 即使上升至 $40 \mathrm{~A} \cdot \mathrm{g}^{-1}$ 时, 比容量仍高 达 $130 \mathrm{mAh} \cdot \mathrm{g}^{-1}$. 此外, 四壳层 $\mathrm{NiS}_{2}$ 中空球还具有优异 的循环稳定性，在 $20 \mathrm{~A} \cdot \mathrm{g}^{-1}$ 下循环 5000 圈，电容保持率 为 $89.96 \%$ (图 $6 \mathrm{a} \sim 6 \mathrm{~d}$ ). 优异的电化学性能得益于 $\mathrm{NiS}_{2}$ 
HoMSs 的多级中空结构提供了更多活性位点, 并缩短 了离子扩散和电子迁移的路径.

2019 年 Qi 等 ${ }^{[114]}$ 以 ZIF67 为前驱体合成出核壳结构 的 $\mathrm{Co}_{3} \mathrm{O}_{4}$ 十二面体, 然后通过硫粉硫化后制备了具有凹 表面的四壳层 $\mathrm{CoS}_{2}$ 中空十二面体(图 6e, 6f). 从 CV 曲线 可以看出, 与 $\mathrm{Co}_{3} \mathrm{O}_{4}$ 相比, $\mathrm{CoS}_{2}$ 表现出更大的 $\mathrm{CV}$ 曲线面 积和峰强, 说明 $\mathrm{CoS}_{2}$ 具有更高的比电容和更快速的氧 化还原动力学行为 $(6 \mathrm{~g})$. 当与活性炭组装成非对称超级 电容器时, 其循环性能极佳, 循环 10000 圈后电容保持 率高达 92.1\%(图 6h). 这主要由于四壳层 $\mathrm{CoS}_{2}$ 中空十二 面体与核壳结构的 $\mathrm{Co}_{3} \mathrm{O}_{4}$ 十二面体相比, 活性材料的利
用率更高，离子和电子传输距离更短，并且具有更好的 结构稳定性.

Chen 等 ${ }^{[115]}$ 以聚合物纳米纤维为软模板合成了均匀 的一维 $\mathrm{M}_{x} \mathrm{Co}_{3-x} \mathrm{~S}_{4}(\mathrm{M}=\mathrm{Ni}, \mathrm{Mn}, \mathrm{Zn})$ 中空管状结构(图 6i, $6 \mathrm{j})$. 由于 $\mathrm{M}_{x} \mathrm{Co}_{3-x} \mathrm{~S}_{4}$ 中空管具有多孔结构, 从而有利于 离子/电子的快速迁移, 并能为反应提供足够的界面, 因 此表现出优异的电化学行为. 如图 $6 \mathrm{k}, 61$ 所示, $\mathrm{MnCo}_{2} \mathrm{~S}_{4}$ 中空纳米管在 $2 \mathrm{~A} \cdot \mathrm{g}^{-1}$ 下, 比电容高达 $1203 \mathrm{~F} \cdot \mathrm{g}^{-1}$, 当电 流密度升至 $20 \mathrm{~A} \cdot \mathrm{g}^{-1}$ 时, 比电容为 $968 \mathrm{~F} \cdot \mathrm{g}^{-1}$, 约是前者 的 $80 \%$. 此外, 即使在 $10 \mathrm{~A} \cdot \mathrm{g}^{-1}$ 下循环 20000 圈, 电容 仅损失 $6 \%$ 左右. a

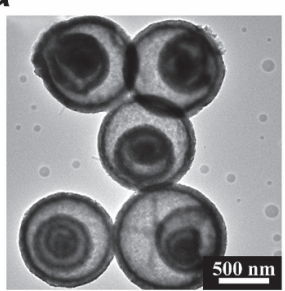

b

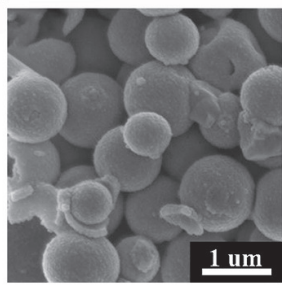

e

e

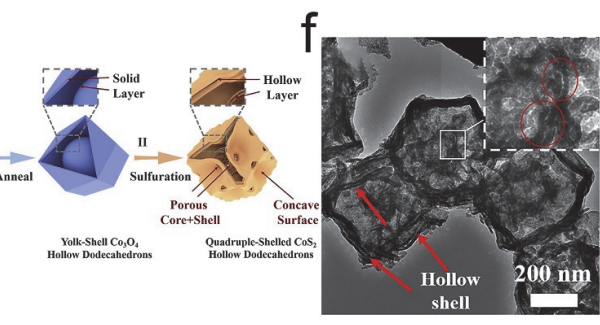

i

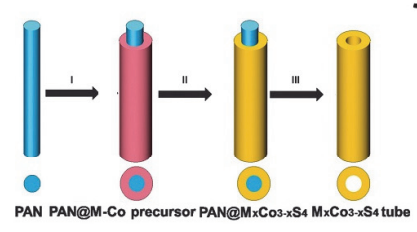

$\mathrm{m}$

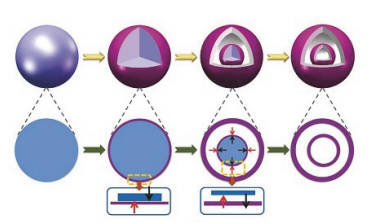

j

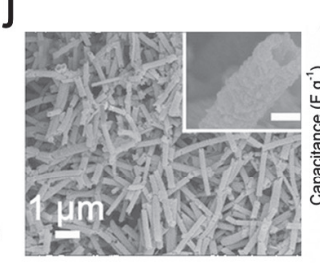

$n$

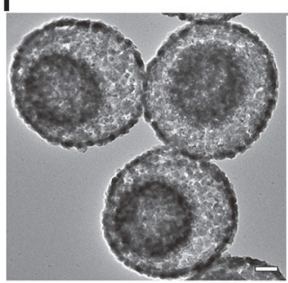

C

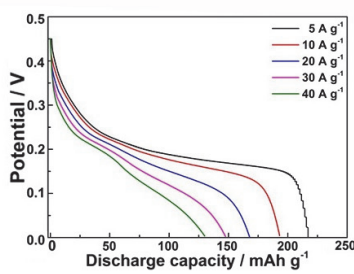

9

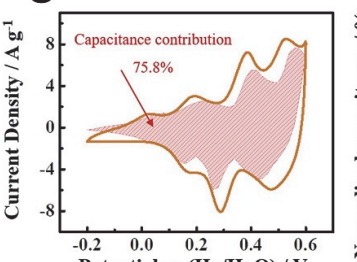

$\mathrm{k}$

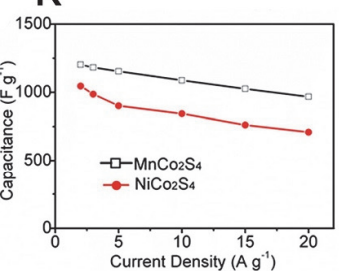

0

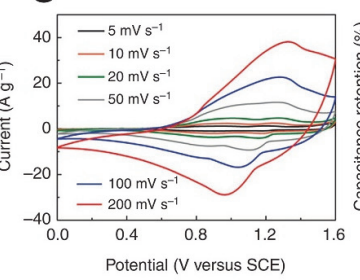

d

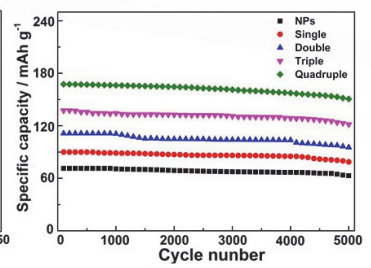

$\mathrm{h}$
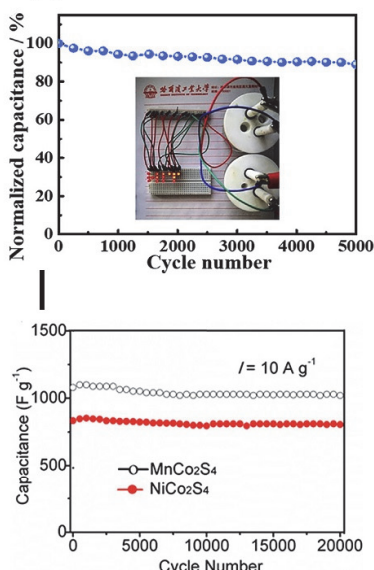

$p$

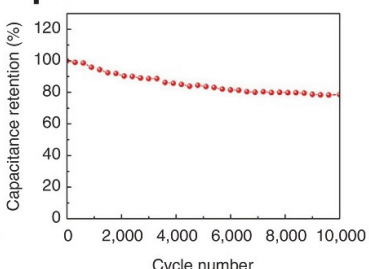

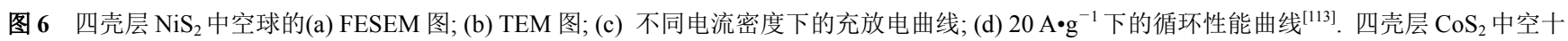
二面体的(e) 合成原理图; (f) TEM 图; (g) 在 $5 \mathrm{mV} \cdot \mathrm{s}^{-1}$ 扫速下, 表面电容储能和扩散控制储能之间容量贡献的差异; (h) 循环性能曲线 ${ }^{[114]}$. $\mathrm{M}_{x} \mathrm{Co}_{3-x} \mathrm{~S}_{4}$ 中空管状结构的(i) 合成机理图; (j) FESEM 图; (k) 不同电流密度下的比电容; (1) $\mathrm{MnCo}_{2} \mathrm{~S}_{4}$ 中空管的循环性能曲线 ${ }^{[115]}$. 双壳层 $\mathrm{NiCo}_{2} \mathrm{~S}_{4}$ 中

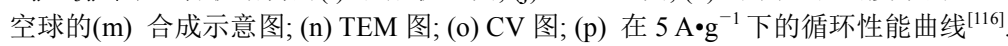

Figure 6 (a) FESEM, (b) TEM images of the quadruple-shelled $\mathrm{NiS}_{2}$ hollow spheres. (c) Galvanostatic charge/discharge curves of quadruple-shelled $\mathrm{NiS}_{2}$ hollow spheres at various current densities. (d) The corresponding cyclic performance for 5000 cycles at a current density of $20 \mathrm{~A} \cdot \mathrm{g}^{-1}[113]$. (e) Schematic illustration for the fabrication of quadruple-shelled $\mathrm{CoS}_{2}$ hollow dodecahedrons electrodes by stepwise synthesis approach. (f) TEM images of quadruple-shelled $\mathrm{CoS}_{2}$ hollow dodecahedrons. (g) Differentiation of the capacity contribution from the surface capacitive energy storage and diffusion-controlled energy storage process at the CV scan rate of $5 \mathrm{mV} \cdot \mathrm{s}^{-1}$. (h) Cycling performance at a constant current density of $5 \mathrm{~A} \cdot \mathrm{g}^{-1}[114]$. (i) Schematic illustration of the formation of $\mathrm{M}_{x} \mathrm{Co}_{3-x} \mathrm{~S}_{4}$ nanotubes by using PAN nanofibers as soft templates. (j) FESEM images of $\mathrm{MnCo}_{2} \mathrm{~S}_{4} \mathrm{HTS}$. (k) Specific capacitance at different current densities, (l) Cycling performance at $10 \mathrm{~A} \cdot \mathrm{g}^{-1}$ of $\mathrm{MnCo}_{2} \mathrm{~S}_{4} \mathrm{HTSs}^{[115]}$. (m) Schematic illustration of the formation process of Ni$\mathrm{Co}_{2} \mathrm{~S}_{4}$ ball-in-ball hollow spheres. (n) TEM images of the $\mathrm{NiCo}_{2} \mathrm{~S}_{4}$ ball-in-ball hollow spheres after annealing in $\mathrm{N}_{2}$. (o) Cyclic voltammetry curves; (p) Cycling performance at $5 \mathrm{~A} \cdot \mathrm{g}^{-1}$ of the $\mathrm{NiCo}_{2} \mathrm{~S}_{4}$ ball-in-ball hollow spheres electrode ${ }^{[116]}$. 
Shen 等 ${ }^{[116]}$ 利用阴离子交换法合成了双壳层的 $\mathrm{NiCo}_{2} \mathrm{~S}_{4}$ 中空球, 其具有优异的赝电容性能(图 $6 \mathrm{~m} \sim 6 \mathrm{p}$ ). 以双壳层 $\mathrm{NiCo}_{2} \mathrm{~S}_{4}$ 中空球为正极, 石墨烯/碳球纳米复合 物薄膜电极为负极, $\mathrm{KOH}$ 为电解液组装成的非对称超级 电容器, 在功率密度为 $476 \mathrm{~W} \cdot \mathrm{kg}^{-1}$ 时, 具有 $42.3 \mathrm{Wh} \cdot$ $\mathrm{kg}^{-1}$ 的高能量密度和超长的循环寿命, 在 $5 \mathrm{~A} \cdot \mathrm{g}^{-1}$ 电流 密度下循环 10000 圈, 电容约为初始值的 $80 \%$. 该混合 超级电容器表现出的高性能主要得益于中空纳米结构 的独特优势.

\section{3 氢氧化物}

在众多的赝电容材料中, 氢氧化镍以其低廉的成 本、储量丰富和较高的理论电容等特点在超级电容器中 得到了广泛的研究 ${ }^{[117,118]}$. Cui 等 ${ }^{[119]}$ 报道了一种层次分 明且平衡良好的大孔、中孔和微孔结构的竹节状石墨碳
纳米纤维. 独特的孔隙结构有助于提高材料的比表面积, 降低离子的传输电阻, 将其组装成柔性全固态超级电容 器, 竹节状石墨碳纳米纤维表现出优异的机械柔韧性和 结构稳定性(图 $7 \mathrm{a} \sim 7 \mathrm{c}$ ). 在 $67 \mathrm{~mA} \cdot \mathrm{cm}^{-3}$ 的电流密度下, 即使循环 10000 次其初始电容保持率仍高达 $96 \%$.

近年来, 多金属氢氧化物由于其性能优于相应的单 金属氢氧化物，在表界面化学能源存储领域具有良好的 应用前景. 层状双氢氧化物因具有组分可调以及高氧化 还原活性等优势引起了广泛的关注 ${ }^{[120]}$. Cao 等 ${ }^{[121]}$ 合成 出一种具有中空纳米笼结构的 Mn-Co 层状双氢氧化物 (Mn-Co LDH, layer double hydroxide). 与普通 $\mathrm{Mn}-\mathrm{Co}$ $\mathrm{LDH}$ 电极相比, 中空纳米笼结构的 Mn-Co LDH 电极具 有更高的比电容和更优异的循环性能(图 $7 \mathrm{~d} \sim 7 \mathrm{f}$ ). 性能 的提高主要归功于中空纳米笼结构的 Mn-Co LDH 增大
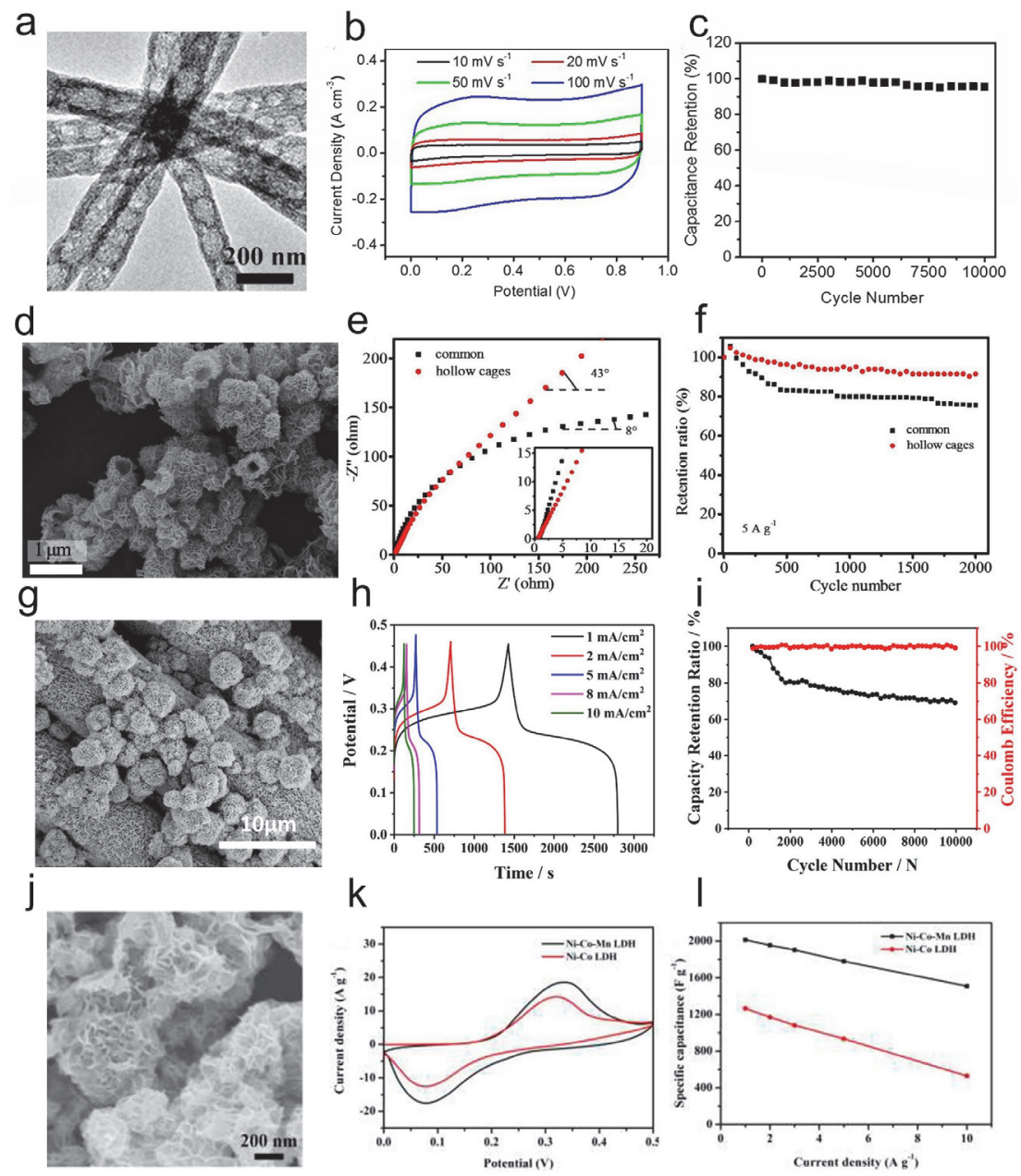

图 7 竹节状石墨碳纳米纤维的(a) TEM 图; 组装成柔性全固态超级电容器的(b) CV 图; (c) 循环性能曲线 ${ }^{[119]}$. Mn-Co LDH 中空纳米笼的 (d) FESEM 图; (e) 能奎斯特曲线; (f) Mn-Co LDH and Mn-Co LDH 中空纳米笼在 $5 \mathrm{~A} \cdot \mathrm{g}^{-1}$ 下的循环性能曲线 ${ }^{[121]}$. H-NiCo LDH@ACC 的(g) SEM 图; (h) 不同电流密度下的充放电曲线; (i) 容量保持率和库伦效率 ${ }^{[122]}$. Ni-Co-Mn LDH 中空纳米笼的(j) SEM 图; Ni-Co-LDH 和 Ni-Co-Mn LDH (k) 在 10 $\mathrm{mV} \cdot \mathrm{s}^{-1}$ 扫速下的 $\mathrm{CV}$ 图; (1) 不同电流密度下的比电容 ${ }^{[124]}$.

Figure 7 (a) TEM images of bamboo-like carbon nanofibers. (b) CV curves, (c) Cycling stability of of a typical all-solid-state flexible supercapacitor ${ }^{[119]}$. (d) FESEM of Mn-Co LDH hollow cages. (e) Nyquist plots of Mn-Co LDH hollow cage electrodes (the inset reveals the high frequency region of the Nyquist plots). (f) Long-term cycling stability of common Mn-Co LDH and Mn-Co LDH hollow cage electrodes at the current density of 5 A $\bullet g^{-1}$ for 2000 cycles $^{[121]}$. (g) SEM images of H-NiCo LDH@ACC. (h) GCD curves of H-NiCo LDH@ACC flexible electrode at various current densities from 1 to 10 $\mathrm{A} / \mathrm{cm}^{2}$. (i) The retention and coulomb efficiency of H-NiCo LDH@ACC ${ }^{[122]}$. (j) SEM images of Ni-Co-Mn LDH hollow nanocage. (k) CV curves of $\mathrm{Ni}-\mathrm{Co}-\mathrm{LDH}$ and Ni-Co-Mn LDH at a scan rate of $10 \mathrm{mV} \cdot \mathrm{s}^{-1}$. (1) specific capacitance versus current density of Ni-Co-LDH and Ni-Co-Mn LDH ${ }^{[124]}$. 
了材料的比表面积, 改善了离子的扩散和电荷的输运. 尽管具有中空纳米结构的层状双氢氧化物表现出良好 的储能性能, 但其电化学性能仍然受到低电导率的限制. 因此, Qian 等 ${ }^{[122]}$ 制备了一种原位生长在酸化碳布上的 中空 $\mathrm{NiCo}$ 层状双氢氧化物(H-NiCo LDH@ACC), 其作 为超级电容器的活性材料表现出较高的比电容和良好 的电化学循环性能 (图 $7 \mathrm{~g} \sim 7 \mathrm{i}$ ). 这种优异的电化学性能 得益于活性材料结合了中空结构特有的优势和碳材料 高导电率的特点.

此外, 掺杂杂原子金属可以有效提高二元层状双氢 氧化物的比电容, 并且能拓宽层间距, 从而减缓充放电 过程产生的机械应力 ${ }^{[123]}$. 基于此, Tao 等 ${ }^{[124]}$ 利用牺牲模 板法成功合成出由三元 Ni-Co-Mn LDH 纳米薄片组装成 的中空纳米笼结构 (图 $7 \mathrm{j} \sim 71$ ). 作为超级电容器电极材 料, 三元 Ni-Co-Mn LDH 中空纳米笼与二元 $\mathrm{Ni}-\mathrm{Co} \mathrm{LDH}$ 中空纳米笼相比, 具有更高的比电容(当电流密度为 1 $\mathrm{A} \cdot \mathrm{g}^{-1}$ 时, 电容为 $\left.1266.2 \mathrm{~F} \cdot \mathrm{g}^{-1}\right)$ 和优异的倍率性能(10 $\mathrm{A} \cdot \mathrm{g}^{-1}$ 时具有 $75 \%$ 的电容保持率). 这主要归因于 Ni-Co-Mn LDH 中空纳米笼的多组分组成、大的比表面 积和多孔结构, 不仅增多了反应位点, 加速了氧化还原 反应，还有利于电子和离子的快速迁移.

\section{4 导电聚合物}

虽然导电聚合物具有成本低、易于合成、导电性好、 工作电压窗口宽、比电容较高等优势, 但其在离子嵌入 和脱出时, 会导致材料溶胀或收缩. 通过优化聚合物的 结构和形貌, 可以缓冲材料的体积变化, 使材料的结构 更稳定, 从而有望提高电容器的整体性能. 聚苯胺 (PANI, Polyaniline) 是最常用的导电聚合物之一. Kang 等 ${ }^{[125]}$ 通过模板法制备了聚苯胺中空微球, 并将其用作 超级电容器电极材料(图 8a). 从恒电流充放电曲线可以 看出聚苯胺中空球在 $1 \mathrm{~mol} \cdot \mathrm{L}^{-1} \mathrm{H}_{2} \mathrm{SO}_{4}$ 溶液中表现出明 显的噟电容行为(图 $8 \mathrm{~b}, 8 \mathrm{c}$ ). 此外, 该超级电容器还具有 较好的稳定性.

2018 年 $\mathrm{Liu}$ 等 ${ }^{[126]}$ 报道了自组装法合成铈离子 $\left(\mathrm{Ce}^{3+}\right)$ 掺杂的聚苯胺中空微球(图 8d). 该研究表明, 掺杂 $\mathrm{Ce}^{3+}$ 的聚苯胺中空球与未掺杂的相比具有更好的电容行为, 在 $1 \mathrm{~mA} \cdot \mathrm{cm}^{-2}$ 电流密度下其比电容为 $248.2 \mathrm{~F} \cdot \mathrm{g}^{-1}$, 而未 掺杂的样品比电容仅为 $201.6 \mathrm{~F} \cdot \mathrm{g}^{-1}$ (图 8e). 此外, $\mathrm{Ce}^{3+}$ 掺 杂显著降低了聚苯胺的电荷转移电阻, 并且直线经过圆 弧后的斜率与理想电容值 $90^{\circ}$ 非常接近, 表现出较好的 电容行为(图 8f). 这主要得益于稀土离子掺杂剂 $\mathrm{Ce}^{3+}$ 作 为氧化还原活性催化剂, 提高了聚苯胺的电导率和比电 容.

为了提高导电聚合物的循环稳定性、比电容和充放 电速率, 研究者们还致力于制备由导电聚合物与碳材料 组成的复合材料 ${ }^{[127]}$. $\mathrm{Ma}$ 等 ${ }^{[128]}$ 设计了一种在还原氧化石 墨烯(rGO)层间插入中空聚苯胺球的三明治结构. 这种 独特的结构能够提高材料的比表面积、吸附更多的离子
和缩短电子的迁移路径(图 $8 \mathrm{~g} \sim 8 \mathrm{i}$ ). 电化学测试结果表 明，在电流密度为 $0.5 \mathrm{~A} \cdot \mathrm{g}^{-1}$ 时，复合材料可以提供 529 $\mathrm{F} \cdot \mathrm{g}^{-1}$ 的高比电容, 并且在 $5 \mathrm{~A} \cdot \mathrm{g}^{-1}$ 下循环 1000 圈后电容 仍能保持 $85 \%$, 远优越于单一的聚苯胺中空球电极材料 性能.

除了中空球状纳米结构外, 中空纳米纤维也受到广 泛关注. Mazinani 等 ${ }^{[129]}$ 合成了一种还原氧化石墨烯修饰 的聚苯胺中空纤维(图 $8 \mathrm{j}$ ). 由于聚苯胺中空纳米纤维结 构优势和 rGO 的三维导电结构协同作用, 使材料在 20 $\mathrm{mV} \cdot \mathrm{s}^{-1}$ 扫速下, 比电容为 $449 \mathrm{~F} \cdot \mathrm{g}^{-1}$, 循环 2000 次后, 电 容损耗率仅为 $16 \%$ (图 $8 \mathrm{k}, 81$ ). 这主要由于 $\mathrm{rGO}$ 可作为 杂化的支架, 保护聚苯胺纳米纤维在长期充放电循环中 不被降解，提高了复合材料的循环稳定性.

\section{6 中空纳米结构在非本征噟电容材料中的应用}

研究表明, 当一些传统的电池型材料尺寸纳米化后 会表现出噟电容行为, 这些非本征赝电容行为可以显著 提高材料的倍率性能 ${ }^{[130-133]}$.

通过调整材料的尺寸和形貌来增加反应位点, 引入 碳材料来降低扩散能垒, 或者在复合材料中创建相界面 来产生更多的活性位点, 可以强化非本征噟电容行为.

为此, 中空纳米结构复合材料在非本征噟电容材料 的应用中得到了广泛的关注. Liang 等 ${ }^{[134]}$ 利用双金属 ZIF-L 为模板，原位合成了双金属硫化物内嵌到中空氮 掺杂碳壳中的复合材料 $\left(\mathrm{Co}_{1} \mathrm{Zn}_{1}-\mathrm{S}(600)\right.$, 图 9a). 电化学 动力学结果分析表明, 复合材料的反应动力学主要由表 面电容控制，这主要得益于纳米尺寸的双金属硫化物中 有大量的相界面(提供了丰富的活性位点和非本征缺陷) 以及中空碳壳结构的高导电性和稳定性(图 9b, 9c). 近 日, Huang 等 ${ }^{[135]}$ 设计了一种在 $\mathrm{Co}_{9} \mathrm{~S}_{8}$ 纳米管内部套有 $\mathrm{Co}_{9} \mathrm{~S}_{8}-\mathrm{C}$ 复合纳米纤维的中空结构, 并将其用作钠离子 电池电极材料. 这种管中纤维的纳米结构具有丰富的晶 界、三个暴露层界面以及碳布线的设计有益于噟电容机 制储能(图 9d). 电化学测试发现, 赝电容存储是钠离子 存储的主导机制，有利于电子与离子的快速传输，因而 材料具有优异的倍率性能(图 9e, 9f). 此外, 中空结构还 有效缓冲了 $\mathrm{Co}_{9} \mathrm{~S}_{8}$ 在循环过程中的体积变化.

\section{7 结论与展望}

总之, 由于中空纳米结构具有的独特的结构优势, 如大的活性比表面积、丰富的活性位点、电极/电解液充 分接触、短的电荷传输路径以及对循环过程中材料体积 膨胀的缓冲作用, 在表界面化学能源存储中具有广阔的 应用前景. 本综述首先讨论了表界面化学能源存储的机 理与挑战, 分析了中空纳米结构的组成和几何特征与表 界面化学储能性能之间的关系. 其次着重介绍了近年来 中空纳米结构在表界面化学能源存储, 尤其是在超级电 容器应用中取得的研究进展. 虽然中空纳米结构有效 

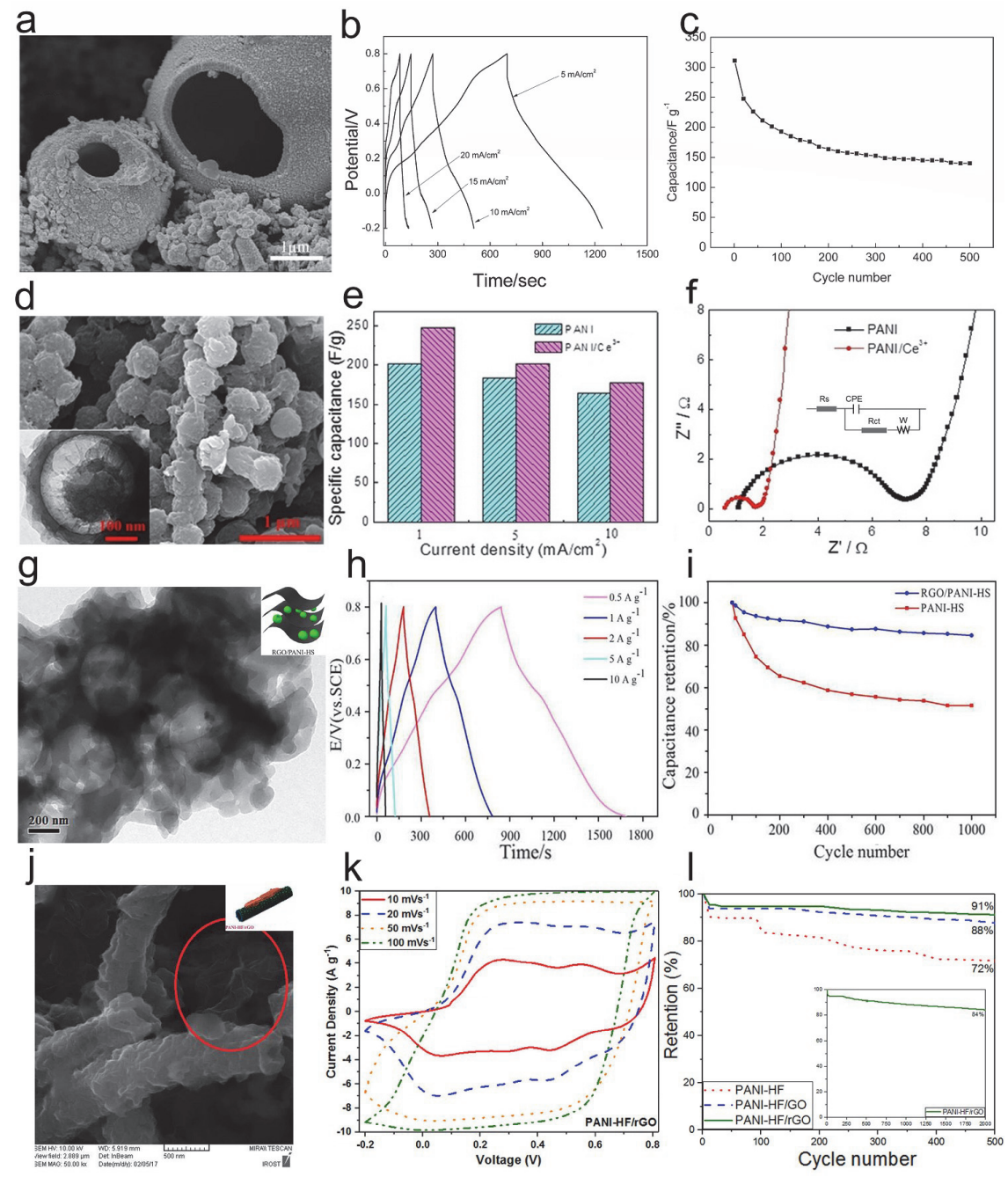

图 8 聚苯胺中空微球的(a) SEM 图; (b) 在 $1 \mathrm{~mol} \cdot \mathrm{L}^{-1} \mathrm{H}_{2} \mathrm{SO}_{4}$ 溶液中的充放电曲线; (c) 在 $10 \mathrm{~mA} / \mathrm{cm}^{2}$ 下的循环寿命 ${ }^{[125]}$. $\mathrm{Ce}^{3+}$ 掺杂的聚苯胺中空微球 (d) SEM 图, 插图为 TEM 图; (e) 不同扫速下的比电容; (f) 能奎斯特曲线 ${ }^{[126]}$. rGO/PANI-HS 复合材料的 (g) TEM 图; (h) 不同电流密度下的充放电 曲线; (i) 循环性能曲线 ${ }^{[128]}$. PANI-HF/GO 的(j) FESEM 图; (k) 不同扫速下的 CV 图; (l) 在 $100 \mathrm{mV} \cdot \mathrm{s}^{-1}$ 扫速下的循环性能曲线 ${ }^{[129]}$.

Figure 8 (a) SEM images of hollow polyaniline microspheres (HMsPANI). (b) Galvanostatic charge-discharge curves of $\mathrm{HMsPANI}^{2} 1 \mathrm{~mol}^{\circ} \mathrm{L}^{-1} \mathrm{H}_{2} \mathrm{SO}_{4}$ solution at different current densities. (c) Cycle life of HMsPANI electrode at discharge current density of $10 \mathrm{~mA} / \mathrm{cm}^{2[125]}$. (d) SEM image of PANI/Ce ${ }^{3+}$ hollow microspheres; inset of (d) is TEM image of PANI/Ce ${ }^{3+}$ hollow microspheres. (e) Specific capacitance versus scan rates. (f) Nyquist plots of PANI and PANI $/ \mathrm{Ce}^{3+}$; inset of (f) is the equivalent circuit model of the EIS plots, here CPE is the constant phase element ${ }^{[126]}$. (g) TEM of rGO/PANI-HS composite. (h) GCD curves of $\mathrm{rGO} / \mathrm{PANI}-\mathrm{HS}$ composite electrode at various current densities from 0.5 to $10 \mathrm{~A} \cdot \mathrm{g}^{-1}$. (i) The cycling life of PANI-HS and

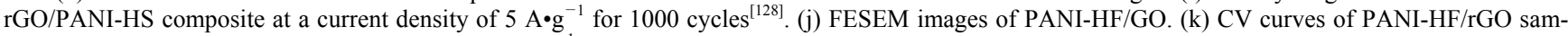
ples at various scan rates of $10,20,50$, and $100 \mathrm{mV}^{-1} \mathrm{~s}^{-1}$. (1) Cycling stability of PANI-HF, PANI-HF/GO, and PANI-HF/rGO after $500 \mathrm{cycles}$ of CV at a scan rate of $100 \mathrm{mV} \cdot \mathrm{s}^{-1}$; Inset: Cycling stability of PANI-HF/rGO after 2000 cycles of $\mathrm{CV}^{[129]}$.

地提高了材料的比电容、倍率性能以及循环稳定性，但 在材料合成方法和化学储能性能方面仍存在一些挑战, 未来的研究还需多方面的努力.

相比于中空单壳层结构, HoMS 单位体积比电容更 高, 且循环稳定性更好. 通过增加 HoMS 壳层数和精确 控制孔结构, 可进一步提高其比电容和循环寿命. 此外, 可以根据不同壳层的导电性、电化学活性和稳定性, 设 计合成具有多种组分结构单元的异质 HoMS, 利用不同 组分之间的协同作用进一步提高中空结构的表界面化 学储能性能, 因而, HoMS 更具有实际应用前景. 值得注 意的是, 虽然 HoMS 具有众多优势, 但其精准的规模化 制备仍存在诸多挑战. 因此, 需要进一步深化对中空纳
米结构形成机理的认识和理解，从而开发出操作更简 便、成本更低且适于工业化生产的合成方法. 目前通过 喷雾干燥等方法，已经可以实现 HoMS 的规模化制备 了. 然而, 喷雾干燥制备的中空材料, 其结构和尺寸的 均匀性较难控制, 有待进一步优化. 其次, 目前 HoMS 的成分主要局限于氧化物、硫化物、氮化物、磷化物等, 对中空纳米结构的合成应用研究还应拓宽到具有独特 电化学优势的异质中空结构材料上. 异质中空结构得益 于不同组分的协同作用，可获得比均质中空结构更广泛 的应用领域和更优异的性能，然而由于其相对更复杂的 合成, 目前异质中空结构还有待进一步研究发展. 另外, 对中空纳米结构可提高表界面储能性能的解释还缺乏 


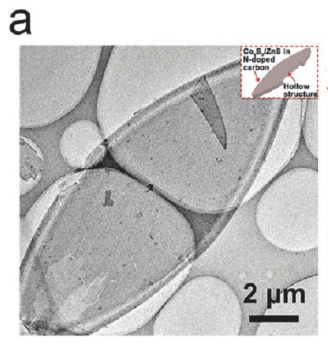

d

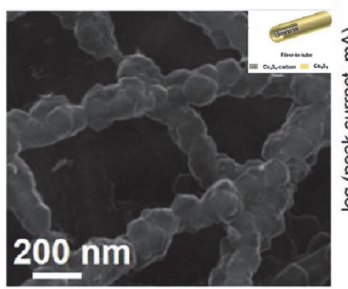

b

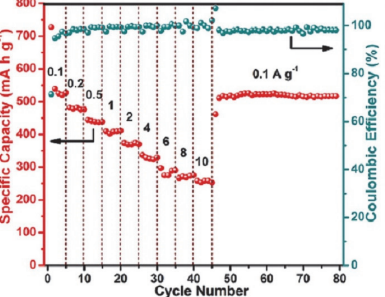

e

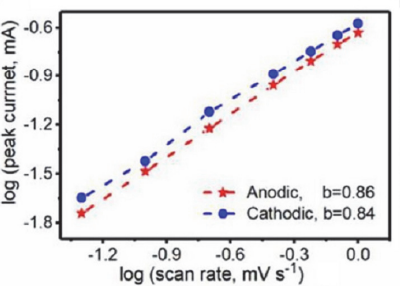

C

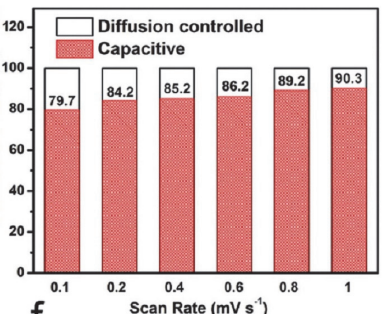

$f$

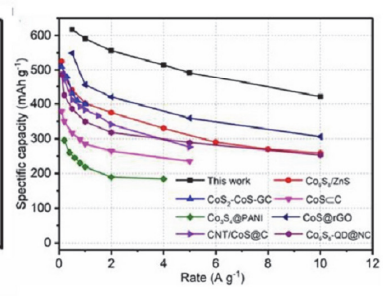

图 9 氮掺杂碳壳复合材料的(a) TEM 图; (b) 倍率性能; (c) 不同扫描率下的赝电容贡献的百分比 ${ }^{[134]} \cdot \mathrm{Co}_{9} \mathrm{~S}_{8}-\mathrm{C} / \mathrm{Co}_{9} \mathrm{~S}_{8}$ 复合材料的(d) FESEM 图; (e) 峰值电流与扫速的关系; (f) 倍率性能 ${ }^{[135]}$.

Figure 9 (a) TEM image of $\mathrm{Co}_{1} \mathrm{Zn}_{1}-\mathrm{S}(600)$ nanosheet, (b) rate capability from 0.1 to $10 \mathrm{~A} \cdot \mathrm{g}^{-1}\left(0.077\right.$ to $\left.7.7 \mathrm{~mA} \cdot \mathrm{cm}^{-2}\right)$, (c) bar chart showing the percent of pseudocapacitive contribution at different scan rates ${ }^{[134]}$. (d) FESEM images of the $\mathrm{Co}_{9} \mathrm{~S}_{8}-\mathrm{C} / \mathrm{Co}_{9} \mathrm{~S}_{8}$ composite, (e) $\log i$ vs $\log v$ plots at oxidation and reduction state, (f) comparison of rate capabilities of the $\mathrm{Co}_{9} \mathrm{~S}_{8}-\mathrm{C} / \mathrm{Co}_{9} \mathrm{~S}_{8}$ composite with other reported $\mathrm{Co}_{9} \mathrm{~S}_{8}$-based electrodes ${ }^{[135]}$.

直接有力的证据, 因此还需要借助于一些先进的原位和 非原位表征技术并结合理论计算来深入地理解结构-性 能关系, 从而更好地指导优化中空纳米结构的设计, 以 进一步提高其表界面化学储能性能. 在众多研究者的共 同努力下, 可以预期中空纳米结构用于表界面化学能源 存储定能迎来更加快速的发展.

\section{作者简介}

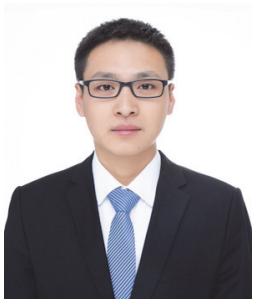

毕如一, 目前在北京科技大学攻读博士学位, 主要的研 究方向为新型二次电池/超级电容器的构建及其关键材料的电 化学性质研究.

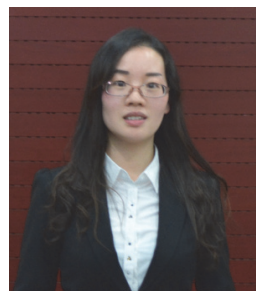

王江艳, 中国科学院过程工程研究所研究员. 2010 年于中 国矿业大学(北京)取得工学学士学位, 2016 年 1 月于中国科学 院大学过程工程研究所获得博士学位. 2016 年 3 月赴美国斯坦 福大学开展博士后研究. 以“微纳米结构材料的设计, 可控合 成及其能源存储应用”为研究核心, 致力于功能化微纳米材料 的设计及可控合成, 并着重研究材料结构与其电池/超级电容 器储能性能的构效关系. 发表 SCI 论文 50 余篇, 其中以第一
作者或共同第一作者总共发表论文 20 余篇，包括 Nat. Energy, Nature Rev. Chem., Sci. Adv., J. Am. Chem. Soc., Nano Lett., Angew. Chem. Int. Ed., Adv. Mater. 等.

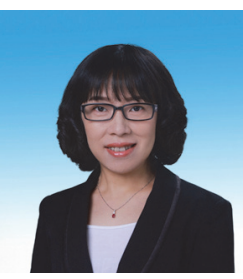

于然波, 教授, 博士生导师. 1994 年毕业于吉林大学化学 系, 1997 年获吉林大学理学硕士学位, 2002 年获得日本国立山 梨大学材料科学专业工学博士学位. 2002 至 2004 年先后作为 日本学术振兴会特别研究员及博士后研究员在日本京都大学 和美国休斯敦大学从事研究工作. 2004 年 4 月起加入北京科技 大学工作. 2005 年北京市“科技新星计划”, 2007 年教育部“新 世纪优秀人才支持计划”, 2016 年获得“日本陶瓷协会中日交 流促进奖”, 2018 年获得中国颗粒学会“自然科学奖”一等奖(第 二完成人), 并担任 Materials Today Energy 期刊国际编委 (Editorial Board Member). 研究方向主要包括无机材料化学和 表面化学, 重点研究微/纳米结构功能材料的发展及其在能量 转换和存储方面的应用. 发表 SCI 论文 180 余篇，包括 Nat. Energy, Adv. Mater, J. Am. Chem. Soc., Angew. Chem. Int. Ed., Chem. Soc. Rev.等，他引 5200 余次，高被引 6 篇. 申请专利 25 项, 已授权 12 项.

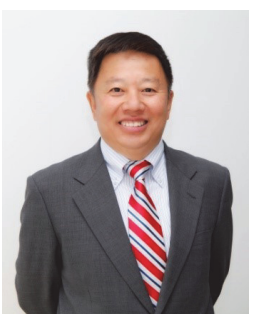

王丹, 研究员, 博士生导师. 1994 年获吉林大学学士学位, 1997 年获吉林大学硕士学位, 2001 年获日本国立山梨大学博 
士学位. 先后在日本京都大学化学研究所等单位从事博士后 研究工作; 2004 年 2 月加入中国科学院过程工程研究所任研究 员. 任高等化学学报与 Chemical Research in Chinses University 杂志的执行主编, Materials Chemistry Frontiers 的副主编, Energy Environmental Science, Matter, Advanced Science 等期刊 的顾问编委. 主要从事无机多功能结构体系的合成化学研究, 包括中空多壳层结构的设计、合成与应用; 二维材料的掺杂与 复合; 多组分高效电极的制备与应用研究. 迄今已在 Nat. Chem., Nat. Energy, Nat. Rev. Chem., Chem. Soc. Rev., Chem, J. Am. Chem. Soc., Angew. Chem. Int. Ed., Adv. Mater. 等期刊发表 SCI 论文 170 余篇, 入选科点唯安(Clarivate Analytics) 2018 年 度与 2019 年度“高被引科学家”名单.

\section{References}

[1] Reddy, A. L. M.; Gowda, S. R.; Shaijumon, M. M.; Ajayan, P. M. Adv. Mater. 2012, 24, 5045.

[2] Chu, S.; Majumdar, A. Nature 2012, 488, 294.

[3] Eshetu, G. G.; Armand, M.; Scrosati, B.; Passerini, S. Angew. Chem., Int. Ed. 2014, 53, 13342.

[4] Peng, Z. K.; Ding, H. M.; Chen, R. F.; Gao, C.; Wang, C. Acta Chim. Sinica 2019, 77, 681. (彭正康, 丁慧敏, 陈如凡, 高超, 汪成, 化 学学报, 2019, 77, 681.)

[5] Liu, C.; Li, F.; Ma, L. P.; Cheng, H. M. Adv. Mater. 2010, 22, E28.

[6] Xu, M.; Yu, Q.; Liu, Z. H.; Lv, J. S.; Lian, S. T.; Hu, B.; Mai, L. Q.; Zhou, L. Nanoscale 2018, 10, 21604.

[7] Yu, Z. N.; Tetard, L.; Zhai, L.; Thomas, J. Y. Energy Environ. Sci. 2015, 8, 702 .

[8] Dubal, D. P.; Ayyad, O.; Ruiz, V.; Gómez-Romero, P. Chem. Soc. Rev. 2015, 44, 1777.

[9] Etacheri, V.; Marom, R.; Elazari, R.; Salitra, G.; Ayrbach, D. Energy Environ. Sci. 2011, 4, 3243.

[10] Jiang, J.; Li, Y. Y.; Liu, J. P.; Huang, X. T.; Yuan, C. Z.; Lou, X. W. Adv. Mater. 2012, 24, 5166.

[11] Simon, P.; Gogotsi, Y.; Dunn, B. Science 2014, 343, 1210

[12] Zhang, L. L.; Zhao, X. Chem. Soc. Rev. 2009, 38, 2520.

[13] Guo, X. T.; Zheng, S. S.; Zhang, G. X.; Xiao, X.; Li, X. R.; Xu, Y. X.; Xue, H. G.; Pang, H. Energy Storage Mater. 2017, 9, 150.

[14] Chen, K. S.; Xu, R.; Luu, N. S.; Secor, E. B.; Hamamoto, K.; Li, Q.; Kim, S.; Sangwan, V. K.; Balla, I.; Guiney, L. M. Nano Lett. 2017, $17,2539$.

[15] Bin, D. S.; Li, Y. M.; Sun, Y. G.; Duan, S. Y.; Lu, Y. X.; Ma, J. M.; Cao, A. M.; Wan, L. J. Adv. Energy Mater. 2018, 8, 1800855.

[16] Wang, Z. B.; Ge, Q. Q.; Shao, J.; Yan, Y. S. J. Am. Chem. Soc. 2009, 131,6910 .

[17] Lai, X. Y.; Halpert, J. E.; Wang, D. Energy Environ. Sci. 2012, 5, 5604.

[18] Qi, J.; Lai, X. Y.; Wang, J. Y.; Tang, H. J.; Ren, H.; Yang, Y.; Jin, Q.; Zhang, L. J.; Yu, R. B.; Ma, G. H.; Su, Z. G.; Zhao, H. J.; Wang, D. Chem. Soc. Rev. 2015, 44, 6749.

[19] Mao, D.; Wan, J. W.; Wang, J. Y.; Wang, D. Adv. Mater. 2019, 31, 1802874.

[20] Wang, J. Y.; Wan, J. W.; Wang, D. Acc. Chem. Res. 2019, 52, 2169.

[21] Bin, D. S.; Lin, X. J.; Sun, Y. G.; Xu, Y. S.; Zhang, K.; Cao, A. M.; Wan, L. J. J. Am. Chem. Soc. 2018, 140, 7127.

[22] Li, B. T.; Huang, J.; Wang, X. J. Chem. Res. Chinese Univ. 2019, 35, 125.

[23] Ren, H.; Yu, R. B.; Wang, J. Y.; Jin, Q.; Yang, M.; Mao, D.; Kisailus, D.; Zhao, H. J.; Wang, D. Nano Lett. 2014, 14, 6679.

[24] Xu, S. M.; Hessel, C. M.; Ren, H.; Yu, R. B.; Jin, Q.; Yang, M.; Zhao, H. J.; Wang, D. Energy Environ. Sci. 2014, 7, 632.

[25] Zhao, X. X.; Wang, J. Y.; Yu, R. B.; Wang, D. J. Am. Chem. Soc. 2018, 140, 17114.

[26] Ren, H.; Yu, R. B.; Qi, J.; Zhang, L. J.; Jin, Q.; Wang, D. Adv. Mater. 2019, 31, 1805754 .

[27] Wang, J. Y.; Tang, H. J.; Wang, H.; Yu, R. B.; Wang, D. Mater. Chem. Front. 2017, 1, 414.

[28] Wang, J. Y.; Cui, Y.; Wang, D. Adv. Mater. 2019, 31, 1801993.

[29] Zhao, X. X.; Yu, R. B.; Tang, H. J.; Mao, D.; Qi, J.; Wang, B.; Zhang, Y.; Zhao, H. J.; Hu, W. P.; Wang, D. Adv. Mater. 2017, 29, 1700550 .
[30] Jiao, C. W.; Wang, Z. M.; Zhao, X. X.; Wang, H.; Wang, J.; Yu, R. B.; Wang, D. Angew. Chem. Int. Ed. 2019, 58, 996.

[31] Wang, X.; Li, Y. B.; Du, L. Y.; Gao, F. J.; Wu, Q.; Yang, L. J.; Chen, Q.; Wang, X. Z.; Hu, Z. Acta Chim. Sinica 2018, 76, 627. (王啸, 李 有涁, 杜玲玉, 高福杰, 吴强, 杨立军, 陈强, 王喜章, 胡征, 化 学学报, 2018, 76, 627.)

[32] Salhabi, E. H. M.; Zhao, J.; Wang, J.; Yang, M.; Wang, B.; Wang, D. Angew. Chem. Int. Ed. 2019, 58, 9078.

[33] Dong, Z. H.; Lai, X. Y.; Halpert, J. E.; Yang, N. L.; Yi, L. X.; Zhai, J.; Wang, D.; Tang, Z. Y.; Jiang, L. Adv. Mater. 2012, 24, 1046.

[34] Dong, Z. H.; Ren, H.; Hessel, C. M.; Wang, J. Y.; Yu, R. B.; Jin, Q.; Yang, M.; Hu, Z. D.; Chen, Y. F.; Tang, Z. Y.; Zhao, H. J.; Wang, D. Adv. Mater. 2014, 26, 905.

[35] Aravindan, V.; Ulaganathan, M.; Madhavi, S. J. Mater. Chem. A 2016, 4, 7538.

[36] Shao, Y. L.; El-Kady, M. F.; Sun, J. Y.; Li, Y. G.; Zhang, Q. H.; Zhu, M. F.; Wang, H. Z.; Dunn, B.; Kanel, R. B. Chem. Rev. 2018, 118, 9233.

[37] Stoller, M. D.; Ruoff, R. S. Energy Environ. Sci. 2010, 3, 1294.

[38] Choi, N. S.; Chen, Z. H.; Freunberger, S. A.; Ji, X. L.; Sun, Y. K.; Amine, K.; Yushin, G.; Nazar, L. F.; Cho, J.; Bruce, P. G. Angew. Chem. Int. Ed. 2012, 51, 9994.

[39] Fuertes, A. B.; Sevilla, M. ChemSusChem 2015, 8, 1049.

[40] Li, B.; Dai, F.; Xiao, Q. F.; Yang, L.; Shen, J. M.; Zhang, C. M.; Cai, M. Energy Environ. Sci. 2015, 9, 102.

[41] Chen, K. F.; Xue, D. F. Chinese J. Chem. 2017, 35, 861.

[42] Wang, C.; Wang, D. Y.; Zheng, S.; Fang, X. Q.; Zhang, W. L.; Tian, Y.; Lin, H. B.; Lu, H. Y.; Jiang, L. Chem. Res. Chin. Univ. 2018, 34, 983.

[43] Senokos, E.; Reguero, V.; Palma, J.; Vilatela, J. J.; Marcilla, R. Nanoscale 2016, 8, 3620 .

[44] Zhao, M. Q.; Zhang, Q.; Huang, J. Q.; Tian, G. L.; Nie, J. Q.; Peng, H. J.; Peng, H. J.; Wei, F. Nat. Commun. 2014, 5, 3410.

[45] Gu, X. Y.; Hong, Y.; Ai, G.; Wang, C. Y.; Mao, W. F. Acta Chim. Sinica 2018, 76, 644. (顾晓瑜, 洪晔, 艾果, 王朝阳, 毛文峰, 化 学学报, 2018, 76, 644.)

[46] Futaba, D. N.; Dai, K. H.; Yamada, T.; Hiraoka, T.; Hayamizu, Y.; Kakudate, Y.; Tanaike, O.; Hatori, H.; Yumura, M.; Iijima, S. Nat. Mater. 2006, 5, 987.

[47] Miller, J. R.; Outlaw, R. A.; Holloway, B. C. Electrochim. Acta 2011, $56,10443$.

[48] Conway, B. E.; Birss, V.; Wojtowicz, J. J. Power Sources 1997, 66, 1.

[49] Yang, Q.; Lu, Z. Y.; Li, T.; Sun, X. M.; Liu, J. F. Nano Energy 2014, 7, 170 .

[50] Guan, B. Y.; Yu, L.; Wang, X.; Song, S. Y.; Lou, X. W. Adv. Mater. 2017, 29, 1605051 .

[51] Wang, W. C.; Zhang, N.; Shi, Z. Y.; Ye, Z. R.; Gao, Q. Y.; Zhi, M. J.; Hong, Z. L. Chem. Eng. J. 2018, 338, 55.

[52] Oliveira, A. H. P.; Oliveira, H. P. J. Power Sources 2014, 268, 45.

[53] Jiang, Y. Q.; Liu, J. P. Energy Environ. Mater. 2019, 2, 30.

[54] Conway, B. E.; Pell, W. G. J. Solid State Electrochem. 2003, 7, 637.

[55] Engelsmann, K.; Lorenz, W. J.; Schmidt, E. J. Electroanal. Chem. 1980, 114,1 .

[56] Herrero, E.; Buller, L. J.; Abruña, H. D. Chem. Rev. 2001, 101, 1897.

[57] Xia, H.; Meng, Y. S.; Yuan, G. L.; Cui, C.; Lu, L. Electrochem. Solid State Lett. 2012, 15, A60.

[58] Hu, C. C.; Chang, K. H.; Lin, M. C.; Wu, Y. T. Nano Lett. 2006, 6, 2690.

[59] He, Q.; Zhang, C.; Li, X.; Wang, X.; Mu, P.; Jiang, J. X. Acta Chim. Sinica 2018, 76, 202. (贺倩, 张崇, 李晓, 王雪, 牟攀, 蒋加兴, 化 学学报, 2018, 76, 202.)

[60] Toupin, M.; Brousse, T.; Bélanger, D. Chem. Mater. 2004, 16, 3184.

[61] Chen, H.; Zhou, S. X.; Chen, M.; Wu, L. M. J. Mater. Chem. 2012 , $22,25207$.

[62] Mastragostino, M.; Arbizzani, C.; Soavi, F. J. Power Sources 2001, 97, 812 .

[63] Zhang, H. H.; Li, J. Y.; Gu, C.; Yao, M. M.; Yang, B.; Lu, P.; Ma, Y. G. J. Power Sources 2016, 332, 413.

[64] Augustyn, V.; Come, J.; Lowe, M. A.; Kim, J. W.; Taberna, P. L.; Tolbert, S. H.; Abruña, H. D.; Simon, P.; Dunn, B. Nat. Mater. 2013, 12,518 .

[65] Tang, H. J.; Wang, J. Y.; Yin, H. J.; Zhao, H. J.; Wang D.; Tang, Z. Y. Adv. Mater. 2015, 27, 1117.

[66] Lukatskaya, M. R.; Mashtalir, O.; Ren, C. E.; Dall' Agnese, Y.; Rozier, P.; Taberna, P. L.; Naguib, M.; Simon, P.; Barsoum, M. W.; 
Gogotsi, Y. Science 2013, 341, 1502.

[67] Brezesinski, T.; Wang, J.; Tolbert, S. H.; Dunn, B. Nat. Mater. 2010, 9, 146.

[68] Choi, C.; Ashby, D. S.; Butts, D. M.; DeBlock, R. H.; Wei, Q. L.; Lau, J.; Dunn, B. Nat. Rev. Mater. 2019.

[69] Salanne, M.; Rotenberg, B.; Naoi, K.; Kaneko, K.; Taberna, P. L.; Grey, C. P.; Dunn, B.; Simon, P. Nat. Energy 2016, 1, 16070.

[70] Okubo, M.; Hosono, E.; Kim, J.; Enomoto, M.; Kojima, N.; Kudo, T.; Zhou, H. S.; Honma, I. J. Am. Chem. Soc. 2007, 129, 7444.

[71] Park, M. S.; Lim, Y. G.; Kim, J. H.; Kim, Y. J.; Cho, J.; Kim, J. S. Adv. Energy Mater. 2011, 1, 1002.

[72] Naoi, K.; Naoi, W.; Aoyagi, S.; Miyamoto, J.; Kamino, T. Acc. Chem. Res. 2013, 46, 1075.

[73] Wang, J. Y.; Tang, H. J.; Zhang, L. J.; Ren, H.; Yu, R. B.; Jin, Q.; Qi, J.; Mao, D.; Yang, M.; Wang, Y.; Liu, P.; Zhang, Y.; Wen, Y. R.; Gu, L.; Ma, G. H.; Su, Z. G.; Tang, Z. Y.; Zhao, H. J.; Wang, D. Nat. Energy 2016, 1, 16050 .

[74] Guan, Q.; Cheng, J. L.; Li, X. D.; Ni, W.; Wang, B. Chinese J. Chem. 2017, 35, 48 .

[75] Zhang, W.; Chi, Z. X.; Mao, W. X.; Lv, R. W.; Cao, A. M.; Wan, L. J. Angew. Chem. Int. Ed. 2014, 126, 12990.

[76] Li, Y. S.; Shi, J. L. Adv. Mater. 2014, 26, 3176.

[77] Wang, Y. P.; Pan, A. Q.; Zhu, Q. Y.; Nie, Z. W.; Zhang, Y. F.; Tang, Y.; Liang, S. Q.; Cao, G. Z. J. Power Sources 2014, 272, 107.

[78] Jia, H. N.; Wang, Z. Y.; Li, C.; Si, X. Q.; Zheng, X. H.; Cai, Y. F.; Lin, J. H.; Liang, H. Y.; Qi, J. L.; Cao, J.; Feng, J. C.; Fei, W. D. J. Mater. Chem. A 2019, 7, 6686.

[79] Shen, L. F.; Yu, L.; Yu, X. Y.; Zhang, X. G.; Lou, X. W. Angew. Chem. Int. Ed. 2015, 54, 1868.

[80] Yu, L.; Hu, H.; Wu, H. B.; Lou, X. W. Adv. Mater. 2017, 29, 1604563.

[81] Zhao, Y. F.; Wei, R.; Jing, H.; Song, Y. F.; Zhang, C. M.; Xiong, D. B.; Gao, F. M.; Wu, J. S.; Xia, Y. Y. ACS Appl. Mater. Interfaces 2015, 7, 1132

[82] Liu, H.; Zhao, D. X.; Gong, G. D.; Zhang, Z. X.; Jia, T.; Chen, H. Z. Acta Chim. Sinica 2019, 40, 18. (刘浩, 赵丁选, 巩国栋, 张祝新, 贾拓, 陈瀚吉, 化学学报, 2019, 40, 18. )

[83] Jiang, H.; Lee, P. S.; Li, C. Z. Energy Environ. Sci. 2013, 6, 41.

[84] Raccichini, R.; Varzi, A.; Passerini, S.; Scrosati, B. Nat. Mater. 2015, 14, 271.

[85] Hu, S.; Rajamani, R.; Yu, X. Appl. Phys. Lett. 2012, 100, 104103.

[86] Yan, T. T.; Xing, G. L.; Ben, T. Acta Chim. Sinica 2018, 76, 366. (闻 婷婷, 邢国龙, 贲腾, 化学学报, 2018, 76, 366.)

[87] Wang, K. W.; Huang, L.; Razzaque, S.; Jin, S. B.; Tan, B. Small 2016, 12, 3134.

[88] Wang, C.; Wang, F. X.; Liu, Z. C.; Zhao, Y. J.; Liu, Y.; Yue, Q.; Zhu, H. W.; Deng, Y. H.; Wu, Y. P.; Zhao, D. Y. Nano Energy 2017, 41, 674.

[89] Chen, L. F.; Lu, Y.; Yu, L.; Lou, X. W. Energy Environ. Sci. 2017, 10, 1777.

[90] Lv, B. J.; Li, P. P.; Liu, Y.; Lin, S. S.; Gao, B. F.; Lin, B. Z. Appl. Surf. Sci. 2018, 437, 169

[91] Li, Z. W.; Zhong, J. L.; Chen, N. N.; Xue, B.; Mi, H. Y. Acta Chim. Sinica 2018, 76, 209. (李志伟, 仲佳亮, 陈楠楠, 薛兵, 米红宇, 化学学报, 2018, 76, 209.)

[92] He, Y.; Xiang, K. X.; Wang, Y. F.; Zhou, W.; Zhu, Y. R.; Xiao, L.; Chen, W. H.; Chen, X. H.; Chen, H.; Cheng, H.; Lu, Z. G. Carbon 2019, 154, 330 .

[93] Cui, C. Y.; Wang, H.; Wang, M.; Ou, X. W.; Wei, Z. X.; Ma, J. M.; Tang, Y. B. Small 2019, 15, 1902659.

[94] Thangavel, R.; Kannan, A. G.; Ponraj, R.; Yoon, G.; Aravindan, V.; Kim, D. W.; Kang, K.; Yoon, W. S.; Lee, Y. S. Energy Stor. Mater. 2020, 25, 702 .

[95] Jia, H. N.; Wang, Z. Y.; Li, C.; Si, X. Q.; Zheng, X. H.; Cai, Y. F.; Lin, J. H.; Liang, H. Y.; Qi, J. L.; Cao, J.; Feng, J. C.; Fei, W. D. J. Mater. Chem. A 2019, 7, 6686.

[96] Hussain, S. K.; Nagaraju, G.; Sekhar, S. C.; Yu, J. S. Energy Stor. Mater. 2020, $27,405$.

[97] Li, L.; Hu, H. L.; Ding, S. J. Chem. J. Chinese Univ. 2018, 39, 2010. (李龙, 胡红利, 丁书江, 高等学校化学学报, 2018, 39, 2010.)

[98] Guan, C.; Liu, X. M.; Ren, W. N.; Li, X.; Cheng, C. W.; Wang, J. Adv. Energy Mater. 2017, 7, 1602391.

[99] Zhao, B.; Huang, S. Y.; Wang, T.; Zhang, K.; Yuen, M. M. F.; Xu, J. B.; Fu, X. Z.; Sun, R.; Wong, C. P. J. Power Sources 2015, 298, 83.

[100] Xu, T.; Meng, Q. H; Fan, Q.; Yang, M.; Zhi, W. Y.; Cao, B. Chinese
J. Chem. 2017, 35, 1575 .

[101] Wang, S.; Fan, X. Y.; Cui, Y.; Gou, L.; Wang, X. G.; Li, D. L. Acta Chim. Sinica 2019, 77, 551. (王珊, 樊小勇, 崔宇, 苟蕾, 王新刚, 李东林, 化学学报, 2019, 77, 551.)

[102] Wang, J. Y.; Tang, H. J.; Ren, H.; Yu, R, B.; Qi, J.; Mao, D.; Zhao, H. J.; Wang, D. Adv. Sci. 2014, 1, 1400011.

[103] Chen, M. J.; Wang, J. Y.; Tang, H. J.; Yang, Y.; Wang, B.; Zhao, H. J.; Wang, D. Inorg. Chem. Front. 2016, 3, 1065.

[104] Wang, C.; Wang, J. Y.; Hu, W. P.; Wang, D. Chem. Res. Chin. Univ $\mathbf{2 0 2 0}, 36,6$.

[105] Deng, Q. L.; Fu, Y. P.; Zhu, C. B.; Yu, Y. Small 2019, 15, 1804884.

[106] Bi, R. Y.; Xu, N.; Ren, H.; Yang, N. L.; Sun, Y. G.; Cao, A. M.; Yu, R. B.; Wang, D. Angew. Chem. Int. Ed. 2020, 132, 4895.

[107] An, C. H.; Wang, Y. J.; Huang, Y. N.; Xu, Y. N.; Jiao, L. F.; Yuan, H. T. Nano Energy 2014, 10, 125.

[108] Wang, Z.; Jia, W.; Jiang, M. L.; Chen, C.; Li, Y. D. Nano Res. 2016, 9, 2026.

[109] Hu, H.; Guan, B. Y.; Lou, X. W. Chem 2016, 1, 102.

[110] Cao, H. L.; Wang, X.; Chen, X.; Liu, H. Y.; Zheng, J. S.; Zhou, W. F. J. Mater. Chem. A 2017, 5, 20729

[111] Hou, L. R.; Shi, Y. Y.; Zhu, S. Q.; Rehan, M.; Pang, G.; Zhang, X. G.; Yuan, C. Z. J. Mater. Chem. A 2017, 5, 133.

[112] Hua, H.; Liu, S. J.; Chen, Z. Y.; Bao, R. Q.; Shi, Y. Y. Hou, L. R.; Pang, G.; Hui, K. N.; Zhang, X. G.; Yuan, C. Z. Sci. Rep. 2016, 6, 20973.

[113] Li, D. W.; Zhao, X. X.; Yu, R. B.; Wang, B.; Wang, H.; Wang, D. Inorg. Chem. Front. 2018, 5, 535.

[114] Jia, H. N.; Wang, Z. Y.; Zheng, X. H.; Cai, Y. F.; Lin, J. H.; Liang, H. Y.; Qi, J. L.; Cao, J.; Feng. J. C.; Li, W. D. Electrochim. Acta 2019 $312,54$.

[115] Chen, Y. M.; Li, Z.; Lou, X. W. Angew. Chem. Int. Ed. 2015, 54, 10521 .

[116] Shen, L. F.; Yu, L.; Wu, H. B.; Yu, X. Y.; Zhang, X. G.; Lou, X. W. Nat. Commun. 2015, 6, 6694.

[117] Sun, H. Y.; Liu, S. W.; Lu, Q. F.; Zhong, H. Y. Mater. Lett. 2014, $128,136$.

[118] Yan, J.; Fan, Z. J.; Sun, W.; Ning, G. Q.; Wei, T.; Zhang, Q.; Zhang, R. F.; Zhi, L. J.; Wei. F. Adv. Funct. Mater. 2012, 22, 2632.

[119] Sun, Y. M.; Sills, R. B.; Hu, X. L.; Seh, Z. W.; Xiao, Y.; Xu, H. H.; Luo, W.; Jin, H. Y.; Xin, Y.; Li, T. Q.; Zhang, Z. L.; Zhou, J.; Cai, W.; Huang, Y. H.; Cui, Y. Nano Lett. 2015, 15, 3899.

[120] Lu, Q.; Chen, Y. P.; Li, W. F.; Chen, J. G. G.; Xiao, J. Q.; Jiao, F. J. Mater. Chem. A 2013, 1, 2331

[121] Wu, N. S.; Low, J. X.; Liu, T.; Yu, J. G.; Cao, S. W. Appl. Surf. Sci. $\mathbf{2 0 1 7}, 413,35$.

[122] Xuan, X. Y.; Qian, M.; Han, L.; Wan, L. J.; Li, Y. Q.; Lu, T.; Pan, L. K.; Niu, Y. P.; Gong, S. Q. Electrochim. Acta 2019, 321, 134710.

[123] Li, X. J.; Du, D. F.; Zhang, Y.; Xing, W.; Xue, Q. Z.; Yan, Z. F. J. Mater. Chem. A 2017, 5, 15460.

[124] Zheng, X. L.; Han, X.; Zhao, X. X.; Qi, J.; Ma, Q. X.; Tao, K.; Han, L. Mater. Res. Bull. 2018, 106, 243.

[125] Tan, Y. T.; Ran, F.; Wang, L. R.; Kong, L. B.; Kang, L. J. Appl Polym. Sci. 2013, 127, 1544.

[126] Li, Z. H.; Hu, J. K.; Li, Y. B.; Liu, J. ChemistrySelect 2018, 3, 6737.

[127] Zhang, J.; Yu, Y.; Liu, L.; Wu, Y. Nanoscale 2013, 5, 3052.

[128] Dai, W. Q.; Ma, L.; Gan, M. Y.; Wang, S. Y.; Sun, X. W.; Wang, H. N.; Zhou, T. Mater. Res. Bull. 2016, 76, 344.

[129] Tabar, F. A.; Sharif, F.; Mazinani, S. Polymer 2018, 154, 80.

[130] Kim, H. S.; Cook, J. B.; Tolbert, S. H.; Dunn, B. J. Electrochem. Soc. 2015, 162, A5083.

[131] Rauda, I. E.; Augustyn, V.; Saldarriaga-Lopez, L. C.; Chen, X. Y.; Schelhas, L. T.; Rubloff, G. W.; Dunn, B.; Tolbert, S. H. Adv. Funct. Mater. 2014, 24, 6717.

[132] Hou, B. H.; Wang, Y. Y.; Guo, J. Z.; Ning, Q. L.; Xi, X. T.; Pang, W. L.; Cao, A. M.; Wang, X. L.; Zhang, J. P.; Wu, X. L. Nanoscale 2018, 10, 9218 .

[133] Wang, J.; Polleux, J.; Lim, J.; Dunn, B. J. Phys. Chem. C 2007, 111 14925.

[134] Fang, G. Z.; Wu, Z. X.; Zhou, J.; Zhu, C. Y.; Cao, X. X.; Lin, T. Q.; Chen, Y. M.; Wang, C.; Pan, A. Q.; Liang, S. Q. Adv. Energy Mater. 2018, 8, 1703155

[135] Li, X. Y.; Li, K. K.; Zhu, S. C.; Fan, K.; Lyu, L. L.; Yao, H. M.; Li, Y. Y.; Hu, J. L.; Huang, H. T.; Mai, Y. W.; Goodenough, J. B. Angew. Chem. Int. Ed. 2019, 58, 6239. 